\title{
Estudio de la movilidad peatonal en un centro urbano: un caso en Costa Rica
}

\author{
Study of the Pedestrian Mobility in an Urban Center: \\ A Case in Costa Rica
}

\author{
Ana Fernández-Garza \\ Henry Hernández-Vega ${ }^{2}$ \\ Universidad de Costa Rica, Costa Rica
}

\begin{abstract}
Resumen
Se analizó la movilidad peatonal en el centro urbano del distrito de Guadalupe de Goicoechea en San José, Costa Rica; el cual incluye: la caracterización de los usuarios y sus flujos, el volumen peatonal y los niveles de servicio peatonal en las aceras estudiadas. Se obtuvieron los volúmenes peatonales mediante contadores automáticos, además se realizaron conteos manuales para evaluar el desempeño del equipo utilizado. Se efectuó una encuesta con el propósito de caracterizar a los usuarios y sus viajes y conocer la percepción acerca de la infraestructura peatonal. El estudio muestra que la población peatonal es muy diversa, aun así, se pueden observar comportamientos como: la disminución en el número de peatones conforme aumenta la edad de la población encuestada después de los 55 años. Los peatones usualmente caminan en la zona de estudio entre cinco y 35 minutos (distancias cortas), un $79 \%$ de los viajes caminado son intermodales siendo el bus el principal modo de transporte relacionado con la movilidad peatonal en el sector. El Tráfico Promedio Diario Semanal (TPDS) para la zona en estudio se encuentra entre los 170 y los 6517 peatones por día. Las aceras permiten el flujo de peatones que transitan por esta, obteniendo un nivel de servicio peatonal adecuado de acuerdo a los análisis de capacidad realizados.
\end{abstract}

Palabras clave: Peatón, movilidad, conteos, aceras, monitoreo peatonal, PLOS

1 Escuela de Ingeniería Civil. Universidad de Costa Rica. Correo electrónico: anagfergar@hotmail.com

2 Investigador, Programa Infraestructura del Transporte. Laboratorio Nacional de Materiales y Modelos Estructurales. Escuela de Ingeniería Civil. Universidad de Costa Rica. Correo electrónico: henry.hernandez@ucr.ac.cr 


\begin{abstract}
We analyzed the pedestrian mobility in the urban center of the district of Guadalupe, in Goicoechea, San José, Costa Rica. The analysis includes the characterization of users and their flows, the pedestrian volume, and the levels of pedestrian service in the sidewalks studied. Pedestrian volumes were obtained through automatic counters; as well, manual counts were performed to evaluate the performance of the equipment used. A survey was conducted to characterize users and their trips and to know the perception about the pedestrian infrastructure. The study showed that the pedestrian population is very diverse, even so behaviors can be observed such as the decrease in the number of pedestrians as the age of the population interviewed increases after 55 years old. Pedestrians usually walk in the study area between five and 35 minutes (short distances); $79 \%$ of the trips are intermodal, being the bus the main mode of transportation associated with pedestrian mobility in the sector. The Daily-Weekly Traffic Average (TPDS) for the study area is between 170 and 6517 pedestrians per day. The sidewalks allow the flow of pedestrians who walk through, obtaining a proper level of pedestrian service according to the capacity analysis performed.
\end{abstract}

Keywords: Pedestrian, mobility, counts, sidewalks, pedestrian monitoring, PLOS

\title{
Introducción
}

El sistema de transporte tiene el objetivo de mover la mayor cantidad de personas al menor costo y tiempo posible de manera segura y confortable. En la actualidad esto no se está cumpliendo en muchas ciudades, al ser estas pensadas para los automóviles, sin priorizar en el transporte público y los medios no motorizados; así que el costo de movilizarnos cada vez es más alto y el tiempo que invertimos cada vez es mayor. De acuerdo con Bull (2003), el gran aumento de la cantidad de vehículos en Latinoamérica ha generado dificultades a los sistemas de transporte en muchas ciudades de la región.

Costa Rica no fue una excepción a como se desarrollaron las ciudades en Latinoamérica, también sufrió una falta de planificación y un crecimiento violento y expansivo, donde se prioriza la construcción de infraestructura para los vehículos. En la ciudad se desarrollan dos ambientes: en el núcleo de estas se tiene un ambiente comercial, financiero y de venta de servicios, mientras que en sus alrededores se destruyó la zona rural para dar paso a la zona residencial (Pérez \& Alvarado, 2004).

Las externalidades negativas del uso del vehículo son cada vez más visibles y las personas más conscientes de estas; principalmente la congestión y el costo de la salud pública, hacen que los medios de transporte alternativos al automóvil sean cada vez más populares (Medina, 2012).

Al promover la movilidad no motorizada en las ciudades se busca que se pueda caminar como forma de transporte y ocio. Se busca una reducción 
Ana Fernández-Garza, Henry Hernández-Vega. Estudio de la movilidad peatonal en un centro urbano: un caso en Costa Rica

en la dependencia del automóvil y de la energía fósil que utiliza, beneficiando a los ciudadanos, al rescatar el espacio público, y al ambiente, al mitigar la contaminación del aire y ruido, con mayores zonas verdes. Los ciudadanos reciben beneficios en cuanto a revitalización de la economía del lugar y en la salud pública en especial se combate la obesidad, la cual con el estrés son las enfermedades del siglo XXI (Ryan \& Lindsey, 2013; Millward, Spinney, \& Scott, 2013). Para lograr esta promoción, es necesario ceder espacio público a los peatones por medio de calles o caminos peatonales y gestionando una compactación de la ciudad.

Las ciudades interesadas en promover la movilidad urbana, inician por la elaboración de un Plan de Movilidad, el cual es la forma de planificar el transporte dándole unidad y estableciendo las metas de todo el sistema de transporte, en donde se privilegie el transporte masivo e intermodal (Torres, 2016).

Un Plan de Movilidad establece: estrategias, regulaciones, presupuestos y recaudación de fondos para satisfacer la necesidad de los ciudadanos de desplazarse; siendo un ciclo en donde se desarrolla el presupuesto, planteamiento de objetivos y prioridades, diagnóstico, elaboración de la estrategia (el plan), implementación de acciones, monitoreo y evaluación de lo realizado (Garricho, Aguilar, Brambila, \& Sabrino, 2014).

Todo plan debe ser replicable, dinámico, adaptable ya que va evolucionado con el cambio de pensar y actuar de la población, así que presenta soluciones a corto plazo, pero pensando y actuando a mediano y largo plazo (Garricho, Aguilar, Brambila, \& Sabrino, 2014).

Es un acuerdo entre el gobierno y la sociedad, ya que ambos van a tener que financiarlo una parte por medio de subsidios e inversión y la otra parte por medio de los impuestos, multas y marchamos los cuales incorporan en la tarifa las externalidades negativas del uso del automóvil. Tanto la sociedad como el estado se ven favorecidos por los resultados del plan (Sepúlveda, y otros, s.f.).

Para medir el éxito de las políticas públicas para promover el transporte sostenible es necesario tener información detallada acerca de los diferentes modos de transporte. Sin embargo; las estadísticas oficiales en Costa Rica se concentran en adquirir información del tránsito vehicular, dejando a un lado a las personas que caminan, así que actualmente existe una escasa información peatonal en cuanto a sus volúmenes, características 
de sus usuarios y las condiciones de la infraestructura. A nivel mundial, la recolección y el estudio de los datos peatonales se ha desarrollado recientemente, así que se tiene una experiencia limitada en monitoreo peatonal y las bases de datos tiene un registro histórico reciente o inexistente. Los datos de volumen peatonal pueden ser muy variables geográficamente, siendo laborioso y costoso conseguirlos (Yang, Ozbay, \& Bartin, 2010).

En los noventas, el monitoreo peatonal y de ciclistas no era un tema de investigación común. Un indicador de la situación es que en el buscador de Google Académico se encontraron solamente 50 resultados. Sin embargo, esto ha cambiado, ya que para el 2011 se encontraron 1600 resultados (Grifin, Nordback, Gotschi, Stolz, \& Kothuri, 2014). Los retos que se presentan respecto al monitoreo peatonal son los siguientes: establecer programas de conteos permanentes, determinar factores mensuales, determinar el número de sitios a evaluar para poder establecer el comportamiento peatonal, lograr caracterizar en detalle al tráfico peatonal y estandarizar la forma de realizar los conteos (Lindsey, Nordback, \& Figliozzi, 2014).

El Ministerio de Obras Públicas y Transporte (Mopt), realiza conteos peatonales y vehiculares de manera manual, en intersecciones, durante periodos pico en respuesta a las solicitudes de semáforos peatonales que se hacen alrededor de todo el país. En estos conteos se cuantifican los peatones que ingresan al área donde el peatón y el vehículo interactúan, es decir, se cuenta cada peatón que ingresa a la intersección, dejando por fuera lo que sucede en las aceras (Umaña, 2015).

El Laboratorio Nacional de Materiales y de Modelos Estructurales (LanammeUCR), ha analizado las facilidades peatonales en autopistas del país, en especial las paradas, ya que cuando se construyeron las autopistas del país no se consideró el crecimiento que se daría al lado de la vía, así que no hay marginales y las paradas están al borde de los carriles de la autopista.

Los informes se concentran en el estudio de los puntos de mayor ocurrencia de atropellos para la justificación de puentes peatonales, igualmente que los conteos realizados por el Mopt se realizan tomando en cuenta la interacción peatón - vehículo, no se ha estudiado por si solo el volumen peatonal. En los informes también se describe la infraestructura peatonal.

Los conteos peatonales se han caracterizado por ser aún más complicados y costosos que los vehiculares, ya que era necesario realizarlos por 
Ana Fernández-Garza, Henry Hernández-Vega. Estudio de la movilidad peatonal en un centro urbano: un caso en Costa Rica

medio manual, pero actualmente la tecnología también ha permitido que se inicie el conteo automatizado.

Los conteos automáticos continuos permiten caracterizar el flujo peatonal por medio de las horas pico, el día típico y analizar como su comportamiento se ve afectado por el clima, el lugar y el día de la semana. Los insumos anteriores permitirán determinar los factores de tránsito peatonal, los cuales permiten expandir los resultados de los conteos de corto plazo, haciendo posible el establecer la cantidad de peatones que transitan por una intersección o por un determinado punto.

En el país, el diseño y construcción de una acera se ha caracterizado por el cumplimiento mínimo de los reglamentos de accesibilidad y usualmente se deja de lado el volumen de peatones que transitan por la acera, así que en muchos casos cumplen con la Ley 7600 para el caso de Costa Rica, pero no se verifica que cuente con la capacidad necesaria para albergar a todos los peatones, ocasionando que en ciertos lugares y especialmente en los cascos urbanos el peatón tenga que invadir la calzada para realizar su recorrido.

En otros casos, las aceras de Costa Rica son inexistentes, se encuentran llenas de obstáculos o no cumplen con la Ley 7600, siendo más angostas, impidiendo el flujo de los peatones, sillas de ruedas o coches (Vega-Padilla \& Hernández-Vega, 2017).

La población peatonal es muy diversa y con necesidades muy variables, las cuales hay que satisfacer (Roberts \& Black, 1997). Los principales retos al diseñar para los peatones es lo poco predecible que pueden ser y que es un grupo muy variado de usuarios (Espinoza, 2015).

Estudiar y entender cada vez más al peatón y conocer los flujos peatonales, brindará a los planificadores y diseñadores criterios para crear, mantener y modificar la infraestructura en la cual el peatón se desenvuelva con comodidad y seguridad, logrando incentivar esta forma de movilidad, con el objetivo de balancear el uso de los medios de transporte, así ayudar a disminuir la demanda de los sistemas más recargados en este momento.

Tener un conocimiento detallado de los peatones, de sus patrones de movilidad y de sus necesidades, permitirá reconstruir ciudades con mayor movilidad y funcionalidad, las cuales permitan al ciudadano elegir el medio de transporte más eficiente para la distancia recorrida. Esto podría permitir, una adecuada promoción los de medios de transporte no motorizados y el transporte público, en detrimento del uso del automóvil. Para lograr lo 
anterior, es necesaria la implementación de políticas públicas y planes de movilidad (Medina, 2012).

\section{Materiales y métodos}

Esta investigación pretende analizar la movilidad peatonal en el Centro de Guadalupe de Goicoechea con el fin de caracterizar los usuarios de las facilidades peatonales, y sus flujos. Recopilando el volumen de peatones y encuestas que permitan conocer la movilidad peatonal, además de establecer los diferentes niveles de servicio peatonales para las aceras en el área analizada.

El presente proyecto se desarrolla dentro de la provincia San José, en el cantón Goicoechea. La provincia San José está conformada por 20 cantones. Goicoechea es el cantón número ocho de la provincia de San José, se encuentra en el extremo noreste del valle central, rodeado por los cantones Vázquez de Coronado y Moravia al norte, San José y Montes de Oca al sur, Cartago al sureste y Tibás al oeste.

El cantón está conformado por siete distritos: Guadalupe, San Francisco, Calle Blancos, Mata de Plátano, Ipís, Rancho Redondo y Purral; cuenta con una superficie aproximada de $38,5 \mathrm{~km}^{2}$.

La zona en estudio se establece en el distrito Guadalupe, el cual es el distrito primero del cantón Goicoechea considerado como el corazón del cantón ya que en este se da la mayor actividad a nivel comercial por su cercanía a la capital. La superficie del distrito Guadalupe es de $2,48 \mathrm{~km}^{2}$ lo cual equivale a un 6,44 \% del territorio del cantón Goicoechea.

Como zona específica de estudio y trabajo dentro del distrito Guadalupe, se establece el sector definido entre avenidas 29 y 33, y entre calles 39 y 67 , el sector definido entre avenidas 33 y 35 , y entre calles 51 y 53 y el sector definido entre avenidas 33 y 35 y entre calles 65 y 67, como se muestra en la Figura 1. Estos sectores se ubican hacia el oeste del distrito. La zona en estudio está limitada por dos intersecciones importantes, en el oeste por la intersección de las rutas nacionales 39 y 218, conocido popularmente como la Rotonda de la Gallito, ubicada diagonal al supermercado Walmart de Guadalupe, al este se tiene la intersección de las rutas nacionales 200 y 218 diagonal a la estación de servicio Total Guadalupe.

Al recorrer la zona en estudio se puede observar que la avenida 31 tiene un uso principalmente de servicios y comercio, mientras que en las 
Ana Fernández-Garza, Henry Hernández-Vega. Estudio de la movilidad peatonal en un centro urbano: un caso en Costa Rica

avenidas 29 y 33 se empieza a tener un uso más residencial. Se encontraron muchas de las casas en abandono, especialmente entre la calle 51 y 47 en la avenida 29. Los principales usos de suelo de la zona en estudio son servicios y comercio.

En este trabajo se considera peatón a las personas que caminan, trotan, corren o transitan por medio de un aparato con ruedas, el cual no sea comprendido en la definición de vehículo automotor, está definición incluye a niños ya sean que van en coche o alzados (Kittelson \& Associates, Inc, Ryan Snyder Associates \& Los Angeles County Bicycle Coallition, 2013). Además, no se considera a los ciclistas como peatones.

Figura 1. Zona de estudio cuadrante oeste del distrito de Guadalupe de Goicoechea

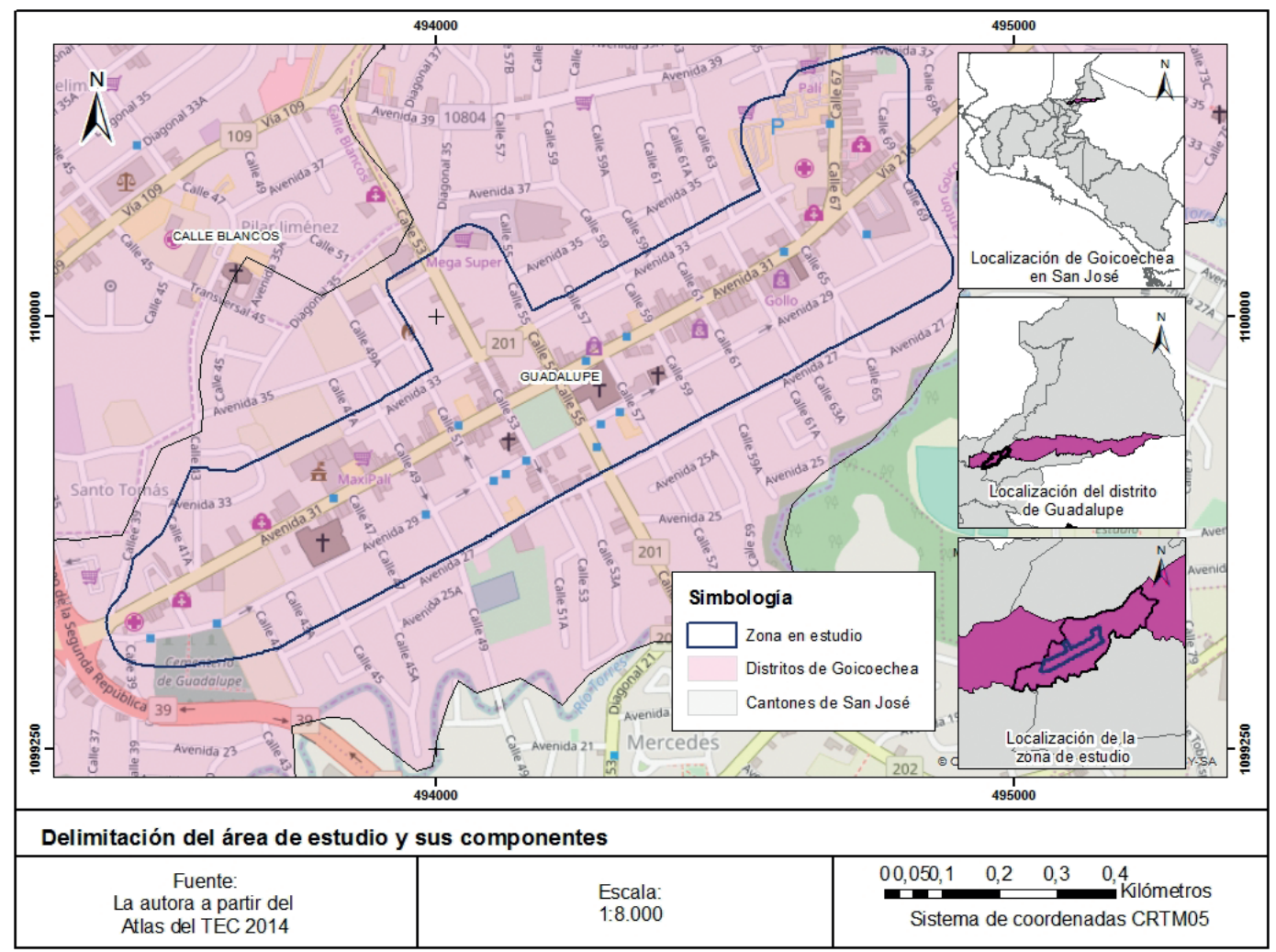


Se realizaron dos tipos de conteo, automático y manual, los cuales se llevaron a cabo en diferentes periodos. Se realizaron conteos automáticos en 46 puntos en la zona en estudio, estos se llevaron a cabo en dos formatos: por periodos y permanente. Los conteos por periodos se desarrollaron en un lapso de tiempo que varía de los ocho días, como mínimo, hasta los 60 días, el cual fue el periodo más largo de los conteos estacionales. El conteo permanente se desarrolló de finales de abril a principio de octubre del 2016 por cinco meses y una semana en total. En siete de los puntos anteriores además se realizaron conteos manuales por dos horas cada uno principalmente en las tardes.

Se contaron todos los peatones que traspasaron una línea imaginaria entre el sensor instalado a un costado de la acera y el borde opuesto de la acera. El dispositivo registra el número de veces que una persona traspasa esa línea imaginaria, sin importar las veces que un mismo peatón la cruzará, por lo que los datos corresponden a la cantidad de viajes realizados por los peatones en cada uno de los puntos analizados.

El equipo utilizado para realizar los conteos automáticos es un contador de sensor infrarrojo pasivo con un lente de alta precisión de la marca Eco-Counter, el sensor se calibra a la temperatura del ambiente y cuando detecta un cambio en el gradiente de temperatura lo relaciona con la presencia de un peatón, registrando los peatones que pasaron frente al sensor cada 15 minutos.

La principal fuente de error del Eco-Counter son los errores sistemáticos de subestimar: cuando los peatones caminan en grupo, en especial cuando va uno a la par del otro o llegan simultáneamente al punto donde se ubica el contador. También puede sobreestimar cuando una persona está parada cercana al sensor, este caso se da en menor medida que el anterior.

Durante el periodo de estudio se colocó un contador automático de manera permanente, en la acera que se encuentra al costado norte de la vía frente a la Municipalidad de Goicoechea, a 34 metros de la esquina de la calle 47 en la avenida 31 . Este sitio se definió como estación permanente de conteo o aforo peatonal.

Se seleccionó el lugar por contar con las mejores condiciones para mitigar los errores del Eco-Counter. Para el análisis se utilizaron los datos del 28 de abril al 16 de octubre. 
Ana Fernández-Garza, Henry Hernández-Vega. Estudio de la movilidad peatonal en un centro urbano: un caso en Costa Rica

En 45 puntos se realizaron conteos temporales y se incluye un resumen de la información del conteo permanente, así que se trabaja con los datos de 46 estaciones distribuidas en la zona de estudio. Cada punto de conteo tiene un número de identificación del 1 al 46, en la Figura 2 se muestra la ubicación de los puntos de conteo.

Para cada punto de conteo se obtuvo la variación del porcentaje del volumen total del día a las diferentes horas de los días entre semana, para el sábado y el domingo. Al tener la curva de cada punto se identificaron las horas pico y se realizó una comparación visual de las similitudes entre curvas para identificar los diferentes grupos.

Se agruparon los puntos en cinco grupos diferentes para el análisis de los días entre semana, en cuatro grupos para el análisis del día sábado y en cinco grupos para el análisis del día domingo.

Figura 2. Ubicación de los estaciones de conteo

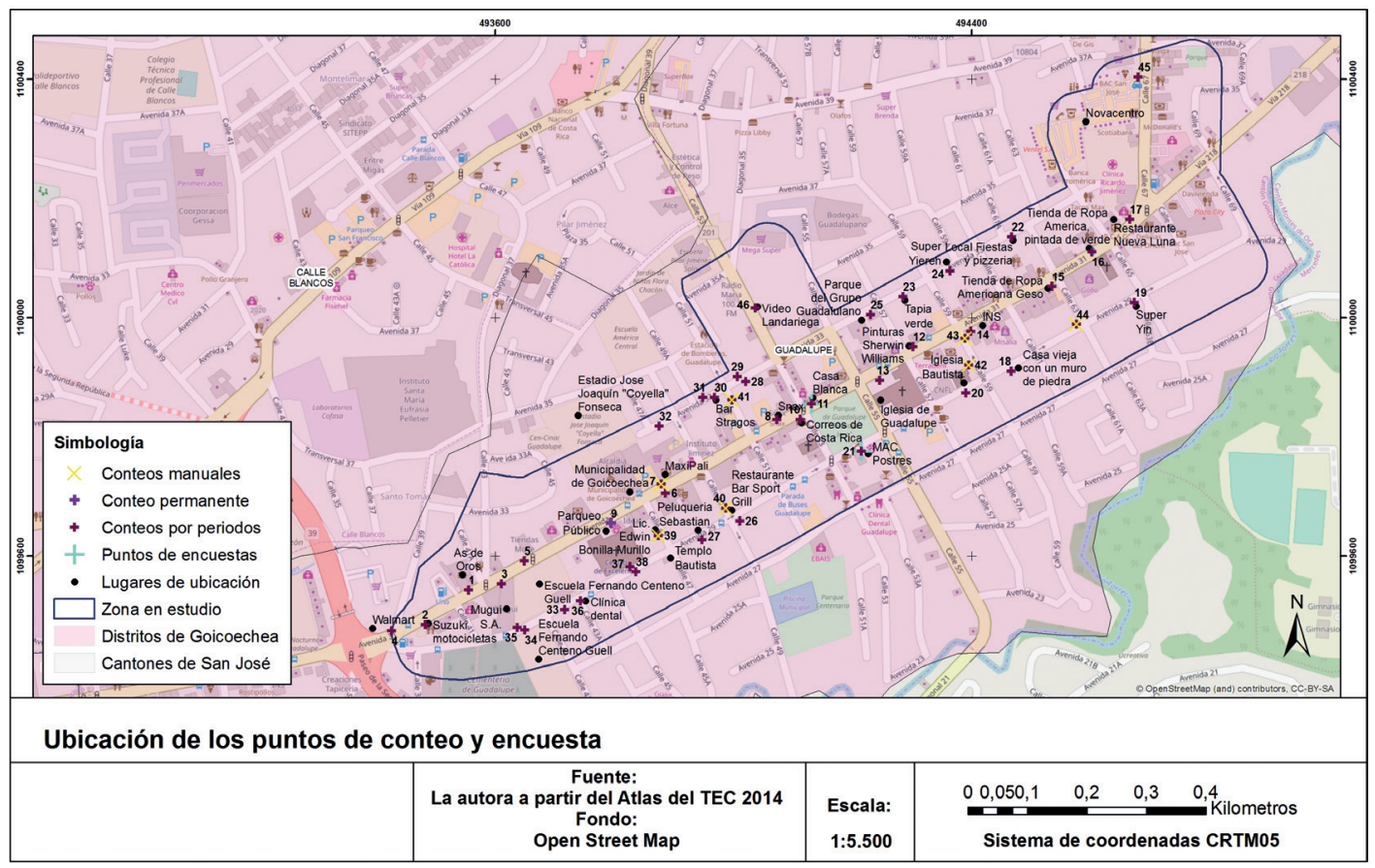


Se realizaron conteos manuales en siete de los puntos y se compararon con el automático. Se calculó la diferencia, considerando que el conteo manual es el más preciso. La diferencia se obtuvo a partir de la siguiente ecuación:

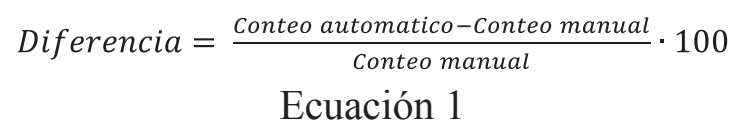

A partir de los resultados obtenidos en los conteos, se definió un tamaño de muestra, cuyo cálculo se explica más adelante, y se procedió a realizar una encuesta. La encuesta es la principal herramienta para entender al peatón, ya que permite determinar las características de los peatones y su opinión acerca de elementos específicos del ambiente vial; se puede preguntar acerca de la infraestructura existente, condiciones deseadas o indeseadas, la precepción de seguridad y los viajes realizados (South Alabama Planning Commission, s.f.).

Es más conveniente realizar las encuestas de manera regional o local, ya que la información que se obtiene describe a las personas y las facilidades de la comunidad, por las cortas distancias que recorren los peatones (Sauter, Pharoah, Tigt, Martinson, \& Wedderburn, 2015).

La metodología de encuesta utilizada fue persona por detención, así que se les pregunta a las personas, que transitaban por los puntos de aforo, si estaban dispuestas a contestar unas preguntas para una investigación. En esta metodología existe un sesgo por participación. Se seleccionaron los puntos que tuvieran el mayor volumen peatonal entre semana aproximadamente. El punto de aforo 11 se ubica en la acera al frente del local de Casa Blanca en la avenida 31 entre las calles 53 y 55 y el punto de aforo 21 se ubica al frente del puesto de postres de McDonald's en la avenida 29 entre las calles 53 y 55, ambos puntos al frente del parque de Guadalupe.

$\mathrm{Al}$ analizar la hora pico en el punto de aforo 11 se encuentra ciertas peculiaridades, la hora pico de la mañana va de las 10:00 a.m. a la 01:00 p.m., la hora pico del medio día de la 01:00 a las 03:00 p.m. y el periodo de la tarde abarca de las 04:00 a las 06:00 p.m. por las condiciones anteriores se puede interpretar que el periodo de mayor flujo peatonal considerado va desde las 10:00 a.m. a las 06:00 p.m. (ver Figura 3 (a)). Se estableció trabajar con un periodo de 12:00 a 05:00 p.m. para determinar la muestra dejando por fuera dos horas en la mañana y una en la tarde, se seleccionó 
Ana Fernández-Garza, Henry Hernández-Vega. Estudio de la movilidad peatonal en un centro urbano:

un caso en Costa Rica

este periodo también porque concuerda con el horario de trabajo de la Unidad de Seguridad Vial del Programa de Infraestructura de Transporte (PITRA) del Laboratorio Nacional de Materiales y Modelos Estructurales (LanammeUCR) de la Universidad de Costa Rica, los cuales colaboraron a realizar las encuestas.

El periodo pico en el punto de aforo 21 es muy fácil de identificar como se muestra en la Figura 3 (b), es de las 04:00 a 6:00 p.m., para aprovechar los recursos disponibles, se decidió trabajar con el periodo de 12:00 a 05:00 p.m. por contar con la ayuda del personal del LanammeUCR para realizar las encuestas.

Figura 3. Volumen horario de peatones en dos ubicaciones

a)

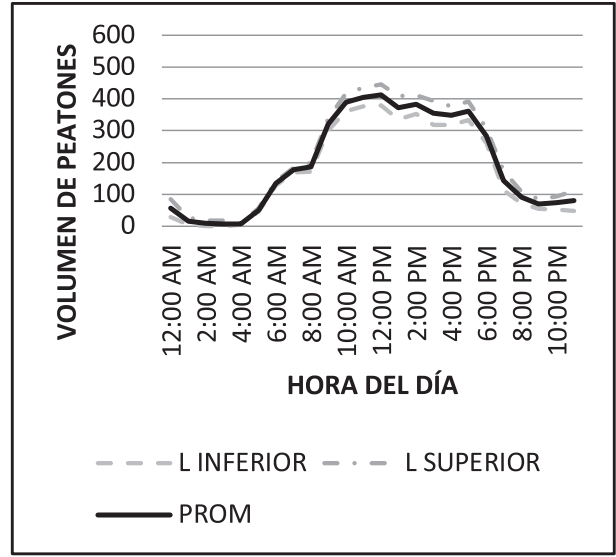

b)

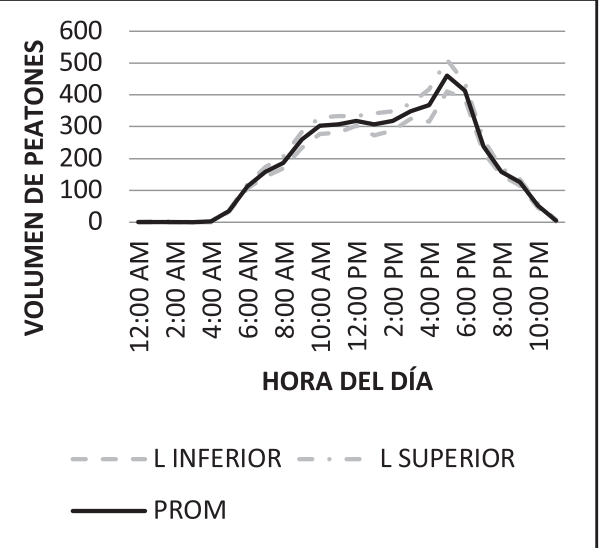

(a) avenida 31 entre las calles 53 y 55, (punto 11) y

(b) avenida 29 entre las calles 53 y 55, (punto 21)

Al contar con los volúmenes y el periodo de análisis se determinó el número de muestra estadísticamente representativa de toda la población peatonal en el punto de aforo. Para obtenerlo se utilizó la ecuación de Willumsen, p: es la proporción de viajeros con destino determinado, siendo $\mathrm{p}=$ 0,5 el valor más conservador2, en donde se usó la población en el periodo de estudio de 12:00 a 5:00 p.m. y población total del día (Ortúzar, 2000). 


$$
n \geq \frac{p(1-p)}{\left(\frac{e}{z}\right)^{2}+\frac{p(1-p)}{N}}
$$

Ecuación 2

Donde:

$\mathrm{p}$ : es la proporción de viajeros con destino determinado, siendo $\mathrm{p}$ $=0,5$ el valor más conservador

e: error asociado que puede ser de $0,1(10 \%)$

z: es la variable normal estándar igual a 1,96 para un nivel de confianza de $95 \%$

$\mathrm{N}$ : tamaño de población

Para el punto 11 se encuestaron 90 personas, en cuanto al punto 21 se logró encuestar en total a 98 personas. Las encuestas se llevaron a cabo los días 17, 21, 28 y 30 de noviembre del 2016 y los días 05, 06 y 07 de diciembre normalmente de la 01:30 a las 05:00 p.m.

La encuesta final se obtuvo después de un proceso de depuración, en donde se obtuvo un borrador de la encuesta la cual se probó en la zona en estudio al realizársela a seis personas, con la experiencia se evalúo la encuesta nuevamente y se formuló la encuesta final.

Además, se realizó un inventario de las aceras existente midiendo el ancho total de la acera y el ancho efectivo, este último se obtiene eliminado todos los obstáculos presentes en la acera siendo el ancho por el cual puede circular el peatón. El ancho mínimo total de acera establecido por ley en Costa Rica es de 1,20 metros.

El ancho efectivo en conjunto con el flujo peatonal permiten calcular el Nivel de servicio peatonal (PLOS, por sus siglas en inglés), el cual es un indicador de la condición de la acera o acceso peatonal, basado en la densidad peatonal y el espacio en la acera. Se utiliza una clasificación alfabética, en donde el nivel óptimo es el A y disminuye hasta el F, en donde se considera que al aumentar el volumen y la densidad peatonal, la velocidad del peatón disminuye, disminuyendo la capacidad de moverse con libertad.

Se calculó los PLOS, tomando el volumen máximo de los intervalos de 15 minutos durante los diferentes días de conteo continuo y con el ancho efectivo de la acera, a partir de la siguiente ecuación: 
Ana Fernández-Garza, Henry Hernández-Vega. Estudio de la movilidad peatonal en un centro urbano: un caso en Costa Rica

$$
P L O S=\frac{Q_{D}}{15 \cdot A_{e}}[\text { peatones } / \mathrm{min} / \mathrm{m}]
$$

Ecuación 3

Donde:

$\mathrm{Q}_{\mathrm{D}}$ : volumen máximo de los intervalos de 15 minutos de conteo [peatones $/ 15 \mathrm{~min}$ ]

$\mathrm{A}_{\mathrm{e}}$ : Ancho efectivo de la acera $[\mathrm{m}]$

Se le aplicaron a los resultados dos categorías la utilizada en el Manual de Capacidad de Carreteras (Transportation Research Board, 2010) y por el manual de la ciudad de Londres (City of London, 2013).

\section{Resultados}

Con el fin de evaluar los datos obtenidos con el contador automático, se realizaron conteos manuales en siete de los puntos en donde se realizaron conteos automáticos. En el Cuadro 1 se presenta el valor obtenido del conteo manual, el automático y la respectiva diferencia relativa. Se observa que la diferencia es usualmente menor al $15 \%$, con excepción de la ubicación del conteo en el punto 44 donde el contador automático registro 17 peatones adicionales a los observados manualmente, con una diferencia relativa del $43 \%$.

Cuadro 1. Resultados obtenidos mediante conteo manual y automático en diferentes sitios

\begin{tabular}{|c|c|c|c|c|c|}
\hline Punto & Fecha & Duración & $\begin{array}{c}\text { Conteo } \\
\text { manual }\end{array}$ & $\begin{array}{c}\text { Contador } \\
\text { automático }\end{array}$ & $\begin{array}{c}\text { Diferencia } \\
\text { Relativa }\end{array}$ \\
\hline 7 & $13 / 05 / 2016$ & $15: 30-17: 30$ & 720 & 770 & $7 \%$ \\
\hline 43 & $19 / 10 / 2016$ & $11: 00-13: 00$ & 89 & 95 & $7 \%$ \\
\hline 39 & $20 / 10 / 2016$ & $07: 05-09: 05$ & 82 & 83 & $1 \%$ \\
\hline 42 & $20 / 10 / 2016$ & $11: 00-13: 00$ & 135 & 154 & $14 \%$ \\
\hline 44 & $21 / 10 / 2016$ & $16: 15-18: 15$ & 40 & 57 & $43 \%$ \\
\hline 41 & $25 / 10 / 2016$ & $15: 45-17: 45$ & 62 & 66 & $6 \%$ \\
\hline 40 & $25 / 10 / 2016$ & $15: 30-17: 30$ & 191 & 166 & $-13 \%$ \\
\hline
\end{tabular}


Para el punto de conteo 9, lugar donde se colocó la estación permanente, se obtuvo una variación del porcentaje del volumen total del día a las diferentes horas del día entre semana, los diferentes puntos se resumen en las siguientes curvas de variación horaria típicos observados que se presenta en la Figura 4.

La información referente al volumen peatonal se utiliza para establecer los patrones de demanda al estudiar las variaciones: horarias, por día de la semana, mensuales y épocas del año, determinado los factores peatonales de expansión (Poapst \& Montufar, 2012).

Las variaciones del volumen de peatones a lo largo de las horas del día, depende de la ubicación y de la actividad que prevalezca en la zona. Para la variación del volumen vehicular, normalmente se diferencia entre los días laborales "entre semana" y los "fines de semana", en donde en los días entre semana presentan un volumen mayor y dos periodos de hora pico uno en la mañana y otro en la tarde. Los fines de semana presentan un comportamiento típico de un periodo de hora pico y menor volumen (Magaña, 2014). Milligan, Poapst y Montufar (2013), mencionan la necesidad de trabajo adicional para identificar los determinantes de los patrones temporales del tráfico peatonal.

Figura 4. Variaciones horarias promedio del volumen peatonal para un día entre semana

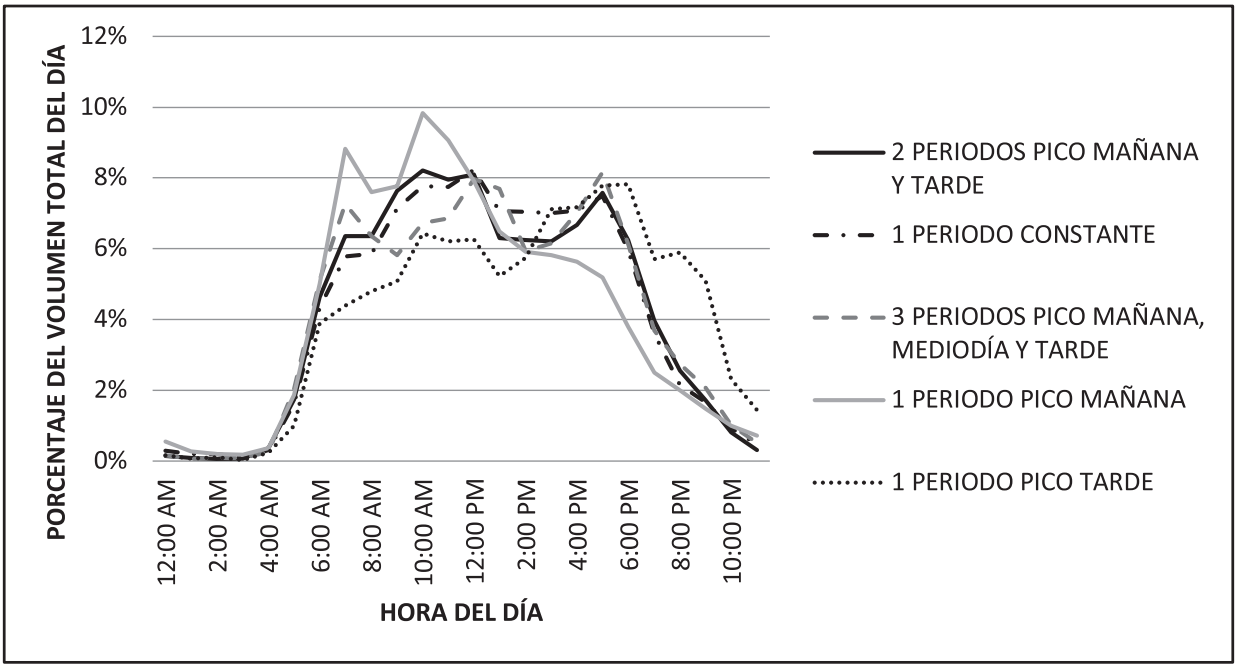


Ana Fernández-Garza, Henry Hernández-Vega. Estudio de la movilidad peatonal en un centro urbano: un caso en Costa Rica

La Figura 5 muestra el volumen diario de todos los días en los que estuvo instalado el contador permanente, en total 171 días. Se puede observar que el volumen de peatones que transitan por el punto nueve va de 650 peatones aproximadamente a los 2400 y que hay una diferencia entre el volumen de días entre semana que tiene un promedio de 1776 peatones, los días sábados tiene un promedio de 1409 y los domingos de 868. Se presentó un dato atípico el día 15 de septiembre; posiblemente la presencia de público presenciando los desfiles, que conmemoran el día de independencia, afectó el sensor ese día en particular.

Al igual que hay variaciones en las horas del día también se encuentra en los días de la semana, como se mencionó anteriormente principalmente entre los días entre semana y los fines de semana; se han encontrado que el volumen de un día entre semana es el doble que el de un día el fin de semana (Figliozzi, Johnson, Monsere, \& Nordback, 2014).

Los conteos de corta duración muestran una fotografía de las condiciones del tráfico peatonal en el momento que son efectuados, así que para utilizar estos datos para estimar condiciones promedio para periodos más largos y ser usados para el análisis, se deben realizar una serie de ajustes para contemplar la variabilidad en el flujo, estos ajustes pueden ser: factores por hora del día, factores por día de la semana y factores por mes del año o estacionales (Magaña, 2014).

Figura 5. Volumen de peatones en la estación permanente (punto 9)

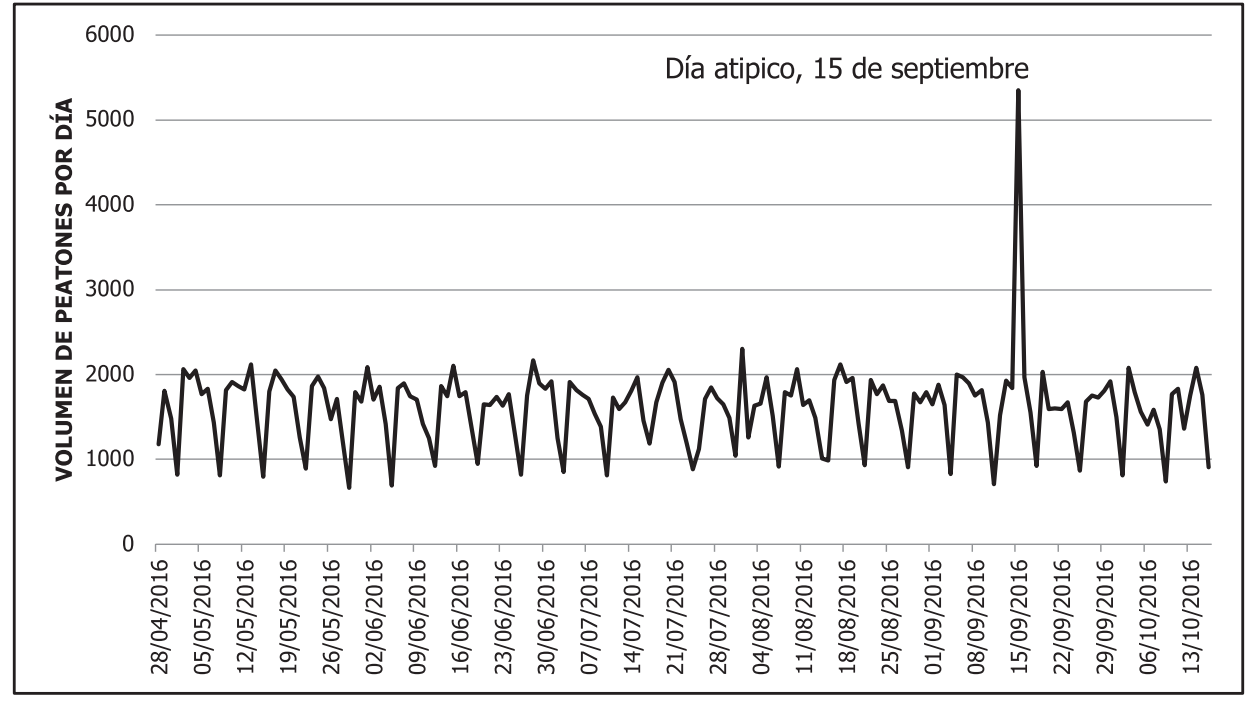


El volumen promedio por día de la semana para cada uno de los seis meses en las que estuvo la estación permanente en funcionamiento, se muestra en la Figura 6.

Figura 6. Volumen peatonal promedio por día de la semana en los diferentes meses del año analizados (punto 9)

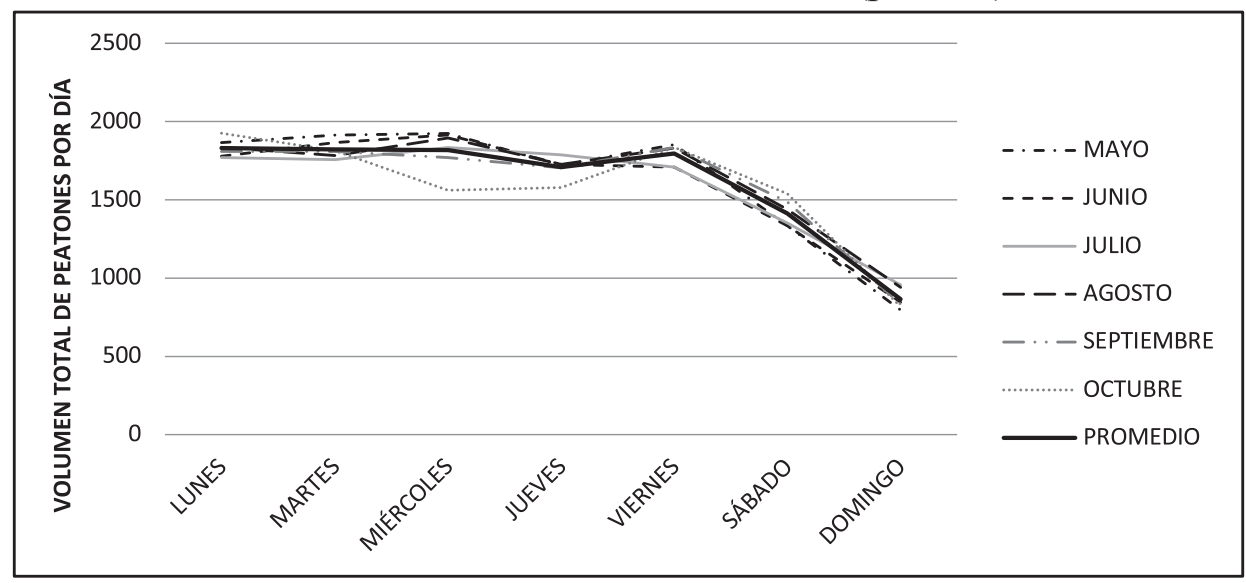

En el Cuadro 2, se presenta la variación del volumen en los meses del año. El Tránsito Promedio Diario Peatonal en el punto nueve es de 1 607 peatones.

Cuadro 2. Volumen promedio diario de peatones por mes (punto 9)

\begin{tabular}{|l|c|}
\hline \multicolumn{1}{|c|}{ Mes del año } & Volumen de peatones diario \\
\hline Mayo & 1630 \\
\hline Junio & 1596 \\
\hline Julio & 1596 \\
\hline Agosto & 1635 \\
\hline Septiembre & 1606 \\
\hline Octubre & 1581 \\
\hline
\end{tabular}

Los factores anteriores también pueden ayudar a determinar un día típico entre semana y en fin de semana, en el cual es preferible realizar los conteos de corta duración en ese lugar o en lugares con condiciones parecidas. Igualmente se pueden realizar conteos de corta duración en una 
semana o varias semanas para observar las variaciones temporales por día de la semana (Figliozzi, Monsere, Nordback, Johnson, \& Blanc, 2014).

El Tráfico Promedio Diario Semanal (TPDS) se analizó en 43 estaciones, en la estación número 18 , no se realizó un conteo que tuviera todos los días de una semana y los puntos 19 y 22 presentan un comportamiento diferente a los demás puntos de estudio. En la Figura 7, se presenta el TPDS para cada punto y su proporción en cuanto viajes direccionales.

Se seleccionaron dos de los puntos de aforo con mayor volumen peatonal entre semana (punto 11 y punto 21) para realizar las encuestas. De acuerdo con las respuestas de los transeúntes, se obtuvieron los resultados que se presentan a continuación, en donde se caracteriza a los peatones, los viajes realizados y la opinión respecto a la infraestructura peatonal.

La encuesta se realizó a 188 personas entre las edades de 13 a los 82 años, de los cuales las mujeres correspondían al 53 \%. En la Figura 8, se muestra la clasificación de los peatones por edad y género. 
Ana Fernández-Garza, Henry Hernández-Vega. Study of the Pedestrian Mobility in an Urban Center: A Case in Costa Rica

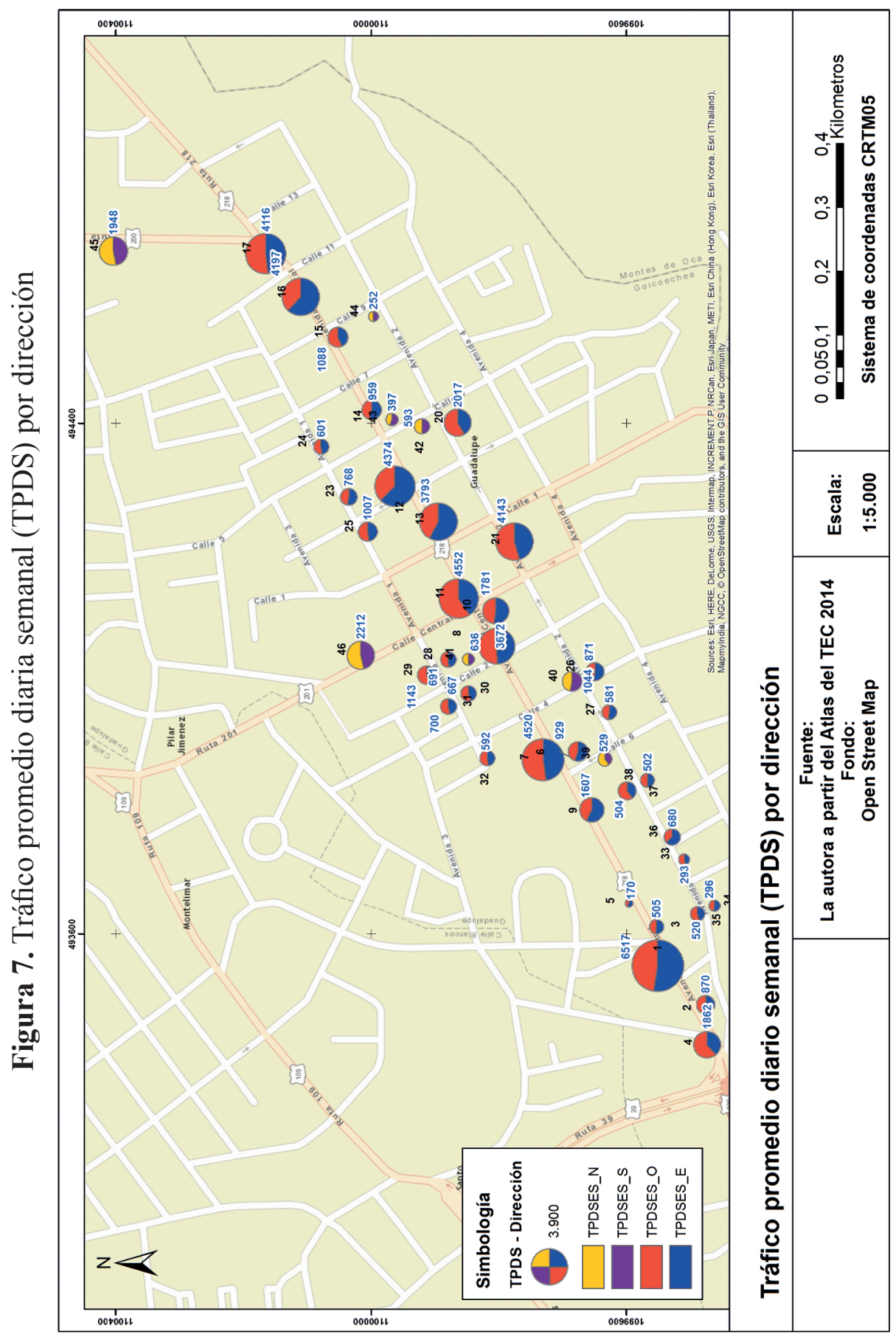


Ana Fernández-Garza, Henry Hernández-Vega. Estudio de la movilidad peatonal en un centro urbano: un caso en Costa Rica

Figura 8. Clasificación de los peatones por edad y género $(n=188)$

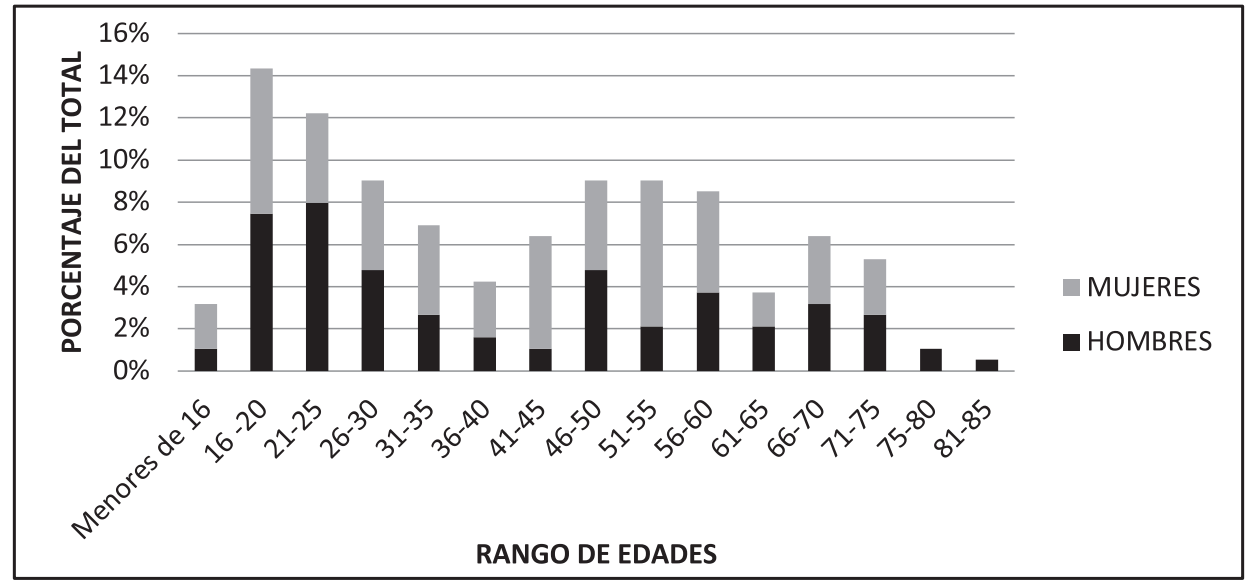

A los peatones se les preguntó el lugar en donde residen en el formato de provincia, cantón y distrito, los 188 encuestados respondieron a la pregunta. El $97 \%$ de los entrevistados vive en la provincia de San José, $4 \%$ en Heredia y un $1 \%$ en Guanacaste. En la Figura 9, se muestra que del $97 \%$ de los peatones que viven en San José un 71 \% viven en el cantón Goicoechea. Del 71 \% de peatones que viven en el cantón Goicoechea, un $36 \%$ viven en Guadalupe, un $6 \%$ en Mata Plátano, un $13 \%$ en Ipís y un $10 \%$ en Purral.

Figura 9. Clasificación de los peatones que residen en (a) la provincia de San José y (b) el cantón de Goicoechea

a)

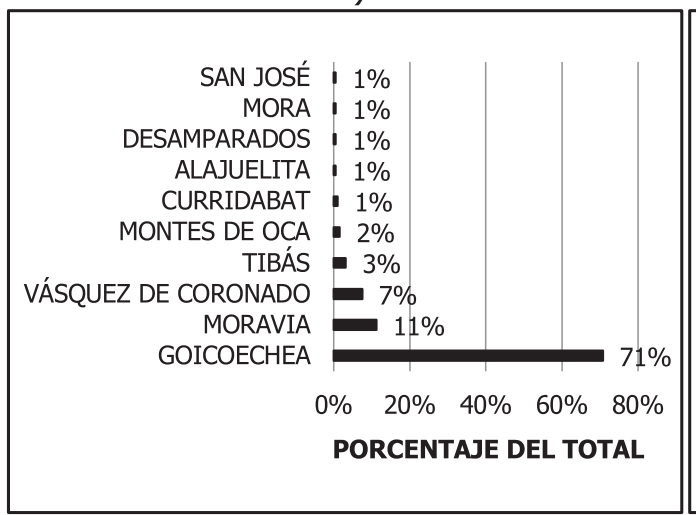

b)

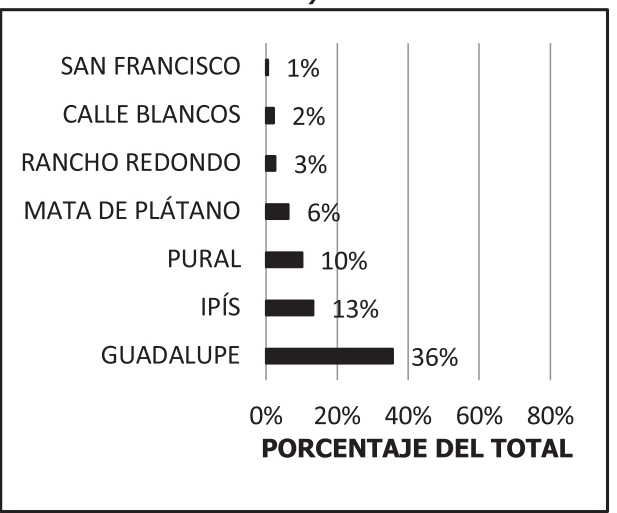


A las 99 personas que respondieron contar con un trabajo, se les preguntó dónde se ubica el trabajo en cuanto a provincia, cantón y distrito. Se obtuvo que un $83 \%$ trabaja en la provincia San José, un $7 \%$ en la provincia Heredia, $1 \%$ trabaja en la provincia Cartago, y $9 \%$ (9 de 99 personas) no respondieron a la pregunta. El detalle de la distribución de personas que trabajan en San José, por cantón se muestra en la Figura 10.

Figura 10. Clasificación de los peatones que laboran de acuerdo a la ubicación del lugar de trabajo en la provincia de San José $(n=99)$

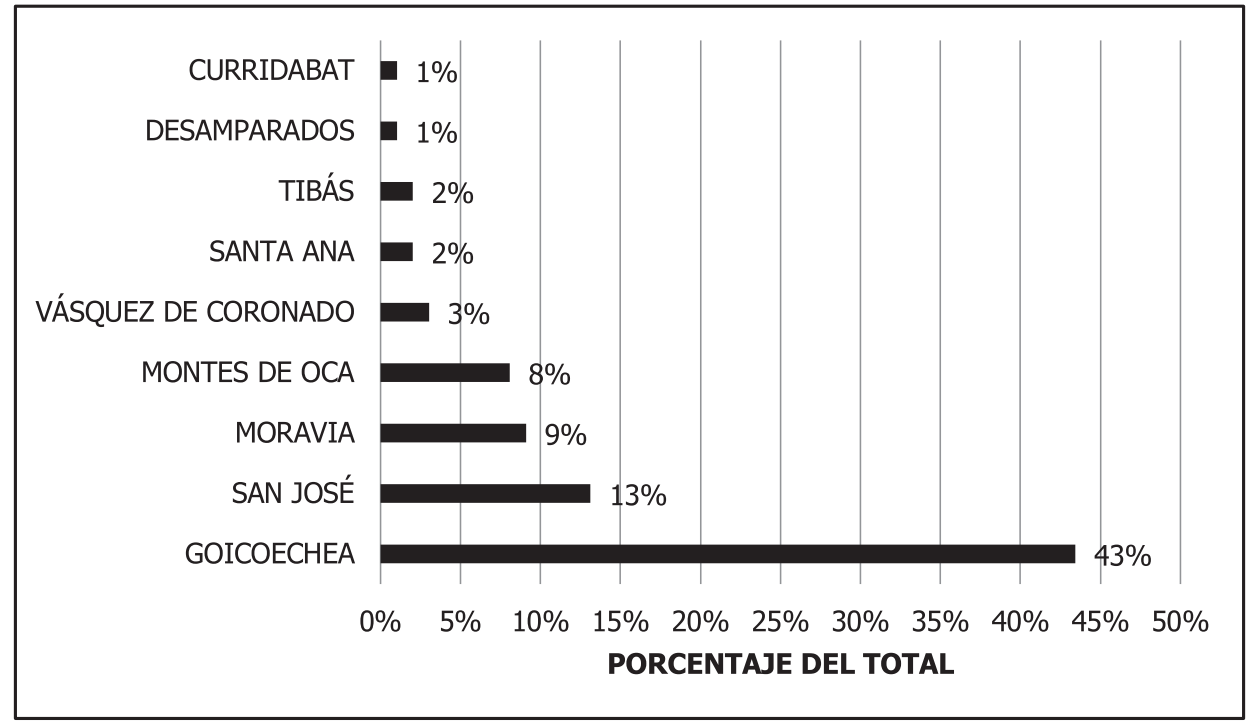

En la Figura 11, se presenta como el motivo que predomina es la realización de algún mandado y el movilizarse desde o al trabajo con un $50 \%$ y un $24 \%$ de las respuestas obtenidas. 
Ana Fernández-Garza, Henry Hernández-Vega. Estudio de la movilidad peatonal en un centro urbano: un caso en Costa Rica

Figura 11. Caracterización de la población peatonal por motivo de viaje $(\mathrm{n}=188)$

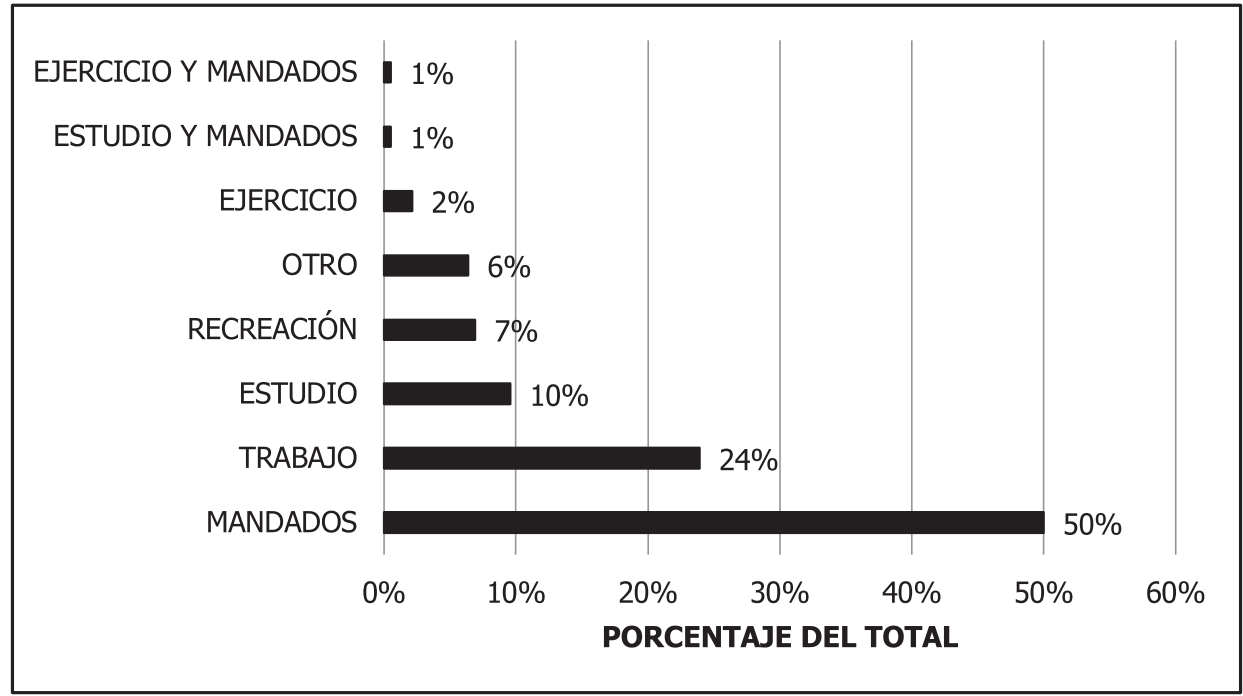

La Universidad de Santa María en Canadá estudió el origen - destino, se encontró que en las mañana el origen es el hogar y el destino es el trabajo, mientras que en la tarde se dan múltiples destinos entre los cuales se encuentran tiendas, paradas de buses y restaurantes, los cuales se realizan en la zona de trabajo y como medio de transporte caminar (Millward, Spinney, \& Scott, 2013).

También en los Estados Unidos se realizó una encuesta nacional acerca de actitudes y comportamiento de los peatones y ciclistas y se encontró que el motivo de viaje más común para los peatones es el realizar diligencias. Se puede percibir la similitud en los resultados de ambas encuestas (Royal \& Miller - Steiger, 2008).

Se les preguntó a los peatones si el viaje realizado al momento de la encuesta es intermodal, a lo cual respondieron 148 peatones (79 \%) que sí y 40 personas $(21 \%)$ realiza el trayecto únicamente caminando. A los peatones que respondieron realizar un viaje intermodal, se les preguntó en cual o cuales otros medios de transportes utilizaron o utilizarán, en la Figura 12 se muestran los resultados. El transporte público y el caminar son sistemas complementarios. El transporte público permite recorrer largas distancias que no son factibles recorrerlas caminado, lo que a su vez 
genera flujo peatonal, ya que los usuarios del transporte público deben usar la red de aceras para llegar a sus destinos intermedios o final (Sauter, Pharoah, Tigt, Martinson, \& Wedderburn, 2015).

Figura 12. Modos que complementan el caminar $(\mathrm{n}=148)$

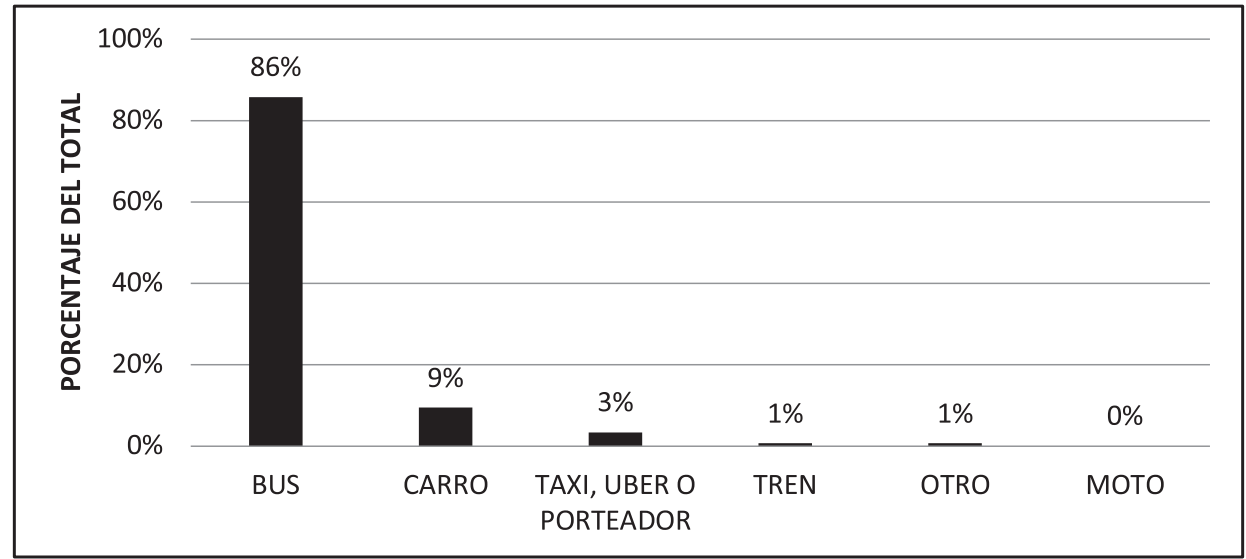

En cuanto a la duración del viaje al calcularlo por origen - destino se presenta que un $49 \%$ camina de 5 a 15 minutos, un $28 \%$ menos de 5 minutos y $10 \%$ entre 16 a 25 minutos, ver Figura 13. La mayoría de los tiempos se encuentran dentro de los rangos reportados en la literatura. 
Ana Fernández-Garza, Henry Hernández-Vega. Estudio de la movilidad peatonal en un centro urbano: un caso en Costa Rica

Figura 13. Tiempo del viaje realizado, calculado a partir del origen destino $(\mathrm{n}=188)$

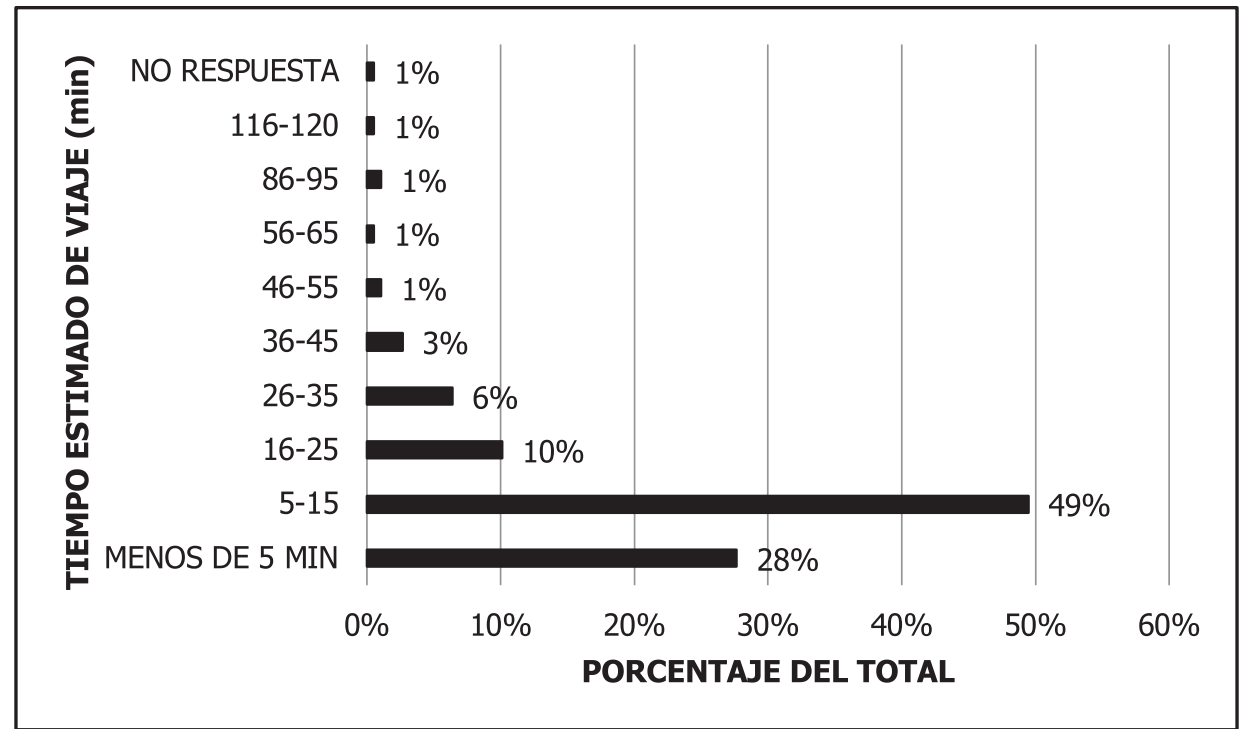

Fuente: Google Maps

Cuadro 3. Tiempo aceptado para hacer un viaje caminando según la literatura

\begin{tabular}{|l|c|c|c|}
\hline \multirow{2}{*}{\multicolumn{1}{|c|}{ Referencia }} & \multicolumn{3}{c|}{ Tiempo (min) } \\
\cline { 2 - 4 } & Mínimo & Promedio & Máximo \\
\hline Ryan \& Lindsey (2013) & 10 & - & 16 \\
\hline Hughes (2002) & - & 9 & - \\
\hline Laing, Turner \& Carr (2009) & 7 & - & 15 \\
\hline Gehl y otros (2009) & 10 & - & 20 \\
\hline Azmi, Karim \& Amin (2012) & 5 & - & 17 \\
\hline Department of Transportation (2016) & 5 & 10 & 20 \\
\hline City of Vancouver, 2015 & 15 & - & 30 \\
\hline Lachapelle \& Noland (2012) & 5 & - & 10 \\
\hline The Gilmore Research Group (2007) & - & 10 & - \\
\hline
\end{tabular}

Se puede recorrer caminado eficientemente distancias cortas, en bicicleta hasta 4000 metros, pero distancias más largas es necesario de otros medios de transporte más veloces y eficientes (Molloy, Kirkpatrick, \& Hull, 2013), así que los sistemas de transporte deben trabajar en conjunto (Ángel, s.f.). 
A las personas encuestadas se les presentaron catorce situaciones relacionadas con el ambiente peatonal y con la acción de caminar, en donde se les preguntó cómo los hacia sentir esas situaciones con respecto a caminar: muy motivado, motivado, no lo afecta, desmotivado, muy desmotivado y no sabe / no aplica.

Aspectos relacionados con árboles, zonas verdes, iluminación, semáforos peatonales y sitios de descanso se podrían encontrar relacionados con una mayor motivación a caminar, tal como se muestra en la Figura 14.

Figura 14. Grado de motivación a caminar bajo diversas situaciones

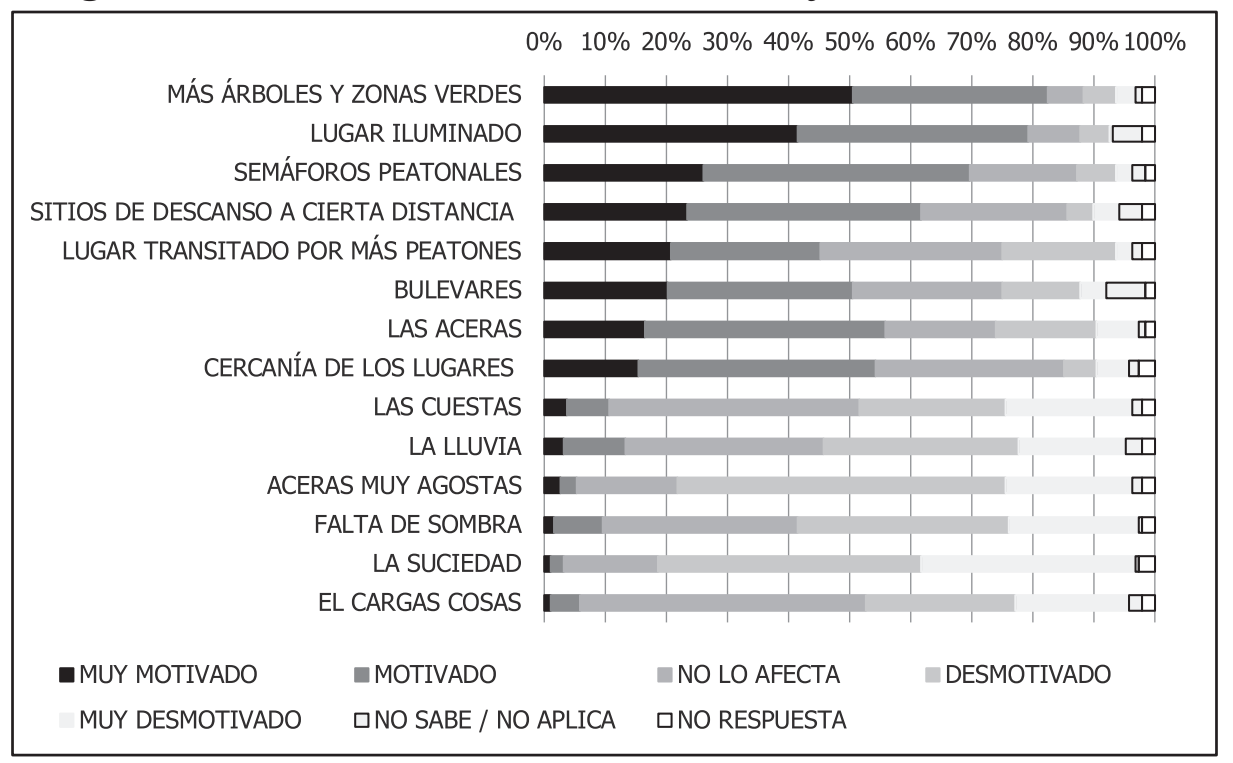

En el Cuadro 4, se presenta el ancho de las aceras en los diferentes puntos de estudio, el ancho efectivo, el PLOS y su calificación alfabética de acuerdo al Manual de Capacidad de Carreteras (indicado como PLOS HCM) (Transportation Research Board, 2010) y por el manual de la ciudad de Londres (indicado como PLOS CL) (City of London, 2013).

El cuadro muestra, que en todos los casos, los niveles de servicio son entre A y B, de acuerdo el PLOS HCM y con el PLOS CL, indicando que no existen niveles de congestión peatonal en las aceras estudiadas. Por otro lado, se encontraron deficiencias en términos de accesibilidad debido a la ausencia de rampas, mal estado de algunas aceras y obstáculos presentes en las mismas. 
Ana Fernández-Garza, Henry Hernández-Vega. Estudio de la movilidad peatonal en un centro urbano: un caso en Costa Rica

Cuadro 4. Nivel de servicio peatonal intervalos de 15 minutos

\begin{tabular}{|c|c|c|c|c|c|c|c|c|c|c|c|}
\hline ID & $\begin{array}{c}\text { Ancho } \\
{[\mathbf{m}]}\end{array}$ & $\begin{array}{c}\text { Ae } \\
{[\mathbf{m}]}\end{array}$ & PLOS & $\begin{array}{c}\text { PLOS } \\
\text { HCM }\end{array}$ & $\begin{array}{c}\text { PLOS } \\
\text { CL }\end{array}$ & ID & $\begin{array}{c}\text { Ancho } \\
{[\mathbf{m}]}\end{array}$ & $\begin{array}{c}\text { Ae } \\
{[\mathbf{m}]}\end{array}$ & PLOS & $\begin{array}{c}\text { PLOS } \\
\text { HCM }\end{array}$ & $\begin{array}{c}\text { PLOS } \\
\text { CL }\end{array}$ \\
\hline 1 & 9,50 & 8,70 & 2,17 & A & A & 24 & 1,25 & 0,45 & 1,92 & A & A + \\
\hline 2 & 1,75 & 1,55 & 3,58 & A & A & 25 & 1,95 & 1,15 & 2,02 & $\mathrm{~A}$ & $\mathrm{~A}+$ \\
\hline 3 & 2,90 & 1,59 & 0,76 & A & A + & 26 & 1,10 & 0,30 & 2,97 & $\mathrm{~A}$ & $\mathrm{~A}+$ \\
\hline 4 & 2,01 & 6,97 & 4,94 & $\mathrm{~A}$ & $\mathrm{~A}$ & 27 & 1,15 & 0,35 & 1,57 & $\mathrm{~A}$ & $\mathrm{~A}+$ \\
\hline 5 & 1,73 & 0,79 & 0,85 & $\mathrm{~A}$ & $\mathrm{~A}+$ & 28 & 1,40 & 0,60 & 2,05 & $\mathrm{~A}$ & $\mathrm{~A}+$ \\
\hline 6 & 1,50 & 2,20 & 1,69 & $\mathrm{~A}$ & $\mathrm{~A}+$ & 29 & 1,25 & 0,45 & 4,53 & $\mathrm{~A}$ & $\mathrm{~A}$ \\
\hline 7 & 1,45 & 6,10 & 9,38 & $\mathrm{~A}$ & $\mathrm{~B}+$ & 30 & 1,75 & 0,95 & 1,18 & $\mathrm{~A}$ & $\mathrm{~A}+$ \\
\hline 8 & 4,60 & 6,55 & 1,72 & $\mathrm{~A}$ & $\mathrm{~A}+$ & 31 & 1,25 & 0,45 & 2,03 & $\mathrm{~A}$ & $\mathrm{~A}+$ \\
\hline 9 & 1,15 & 5,07 & 14,49 & $\mathrm{~A}$ & $\mathrm{~B}$ & 32 & 1,25 & 0,45 & 3,52 & $\mathrm{~A}$ & $\mathrm{~A}$ \\
\hline 10 & 2,80 & 6,33 & 3,17 & $\mathrm{~A}$ & $\mathrm{~A}$ & 33 & 1,40 & 0,80 & 1,57 & $\mathrm{~A}$ & $\mathrm{~A}+$ \\
\hline 11 & 1,20 & 6,13 & 15,33 & $\mathrm{~A}$ & $\mathrm{~B}-$ & 34 & 1,50 & 0,90 & 1,33 & $\mathrm{~A}$ & $\mathrm{~A}+$ \\
\hline 12 & 1,65 & 5,98 & 7,03 & $\mathrm{~A}$ & $\mathrm{~A}-$ & 35 & 3,10 & 2,30 & 0,62 & $\mathrm{~A}$ & $\mathrm{~A}+$ \\
\hline 13 & 3,60 & 9,07 & 3,24 & $\mathrm{~A}$ & $\mathrm{~A}$ & 36 & 4,65 & 3,85 & 1,25 & $\mathrm{~A}$ & $\mathrm{~A}+$ \\
\hline 14 & 1,00 & 0,69 & 3,47 & $\mathrm{~A}$ & $\mathrm{~A}$ & 37 & 1,25 & 0,45 & 1,44 & $\mathrm{~A}$ & $\mathrm{~A}+$ \\
\hline 15 & 2,40 & 3,64 & 2,28 & $\mathrm{~A}$ & $\mathrm{~A}+$ & 38 & 4,92 & 4,32 & 1,50 & $\mathrm{~A}$ & $\mathrm{~A}+$ \\
\hline 16 & 2,10 & 1,30 & 5,71 & $\mathrm{~A}$ & $\mathrm{~A}$ & 39 & 2,60 & 1,80 & 0,85 & $\mathrm{~A}$ & $\mathrm{~A}+$ \\
\hline 17 & 2,10 & 1,30 & 5,43 & $\mathrm{~A}$ & $\mathrm{~A}$ & 40 & 1,70 & 0,90 & 1,92 & $\mathrm{~A}$ & $\mathrm{~A}+$ \\
\hline 18 & 1,25 & 0,65 & 7,15 & $\mathrm{~A}$ & $\mathrm{~A}-$ & 41 & 1,80 & 1,00 & 3,81 & $\mathrm{~A}$ & $\mathrm{~A}$ \\
\hline 19 & - & - & - & - & - & 42 & 1,27 & 0,47 & 3,31 & $\mathrm{~A}$ & $\mathrm{~A}$ \\
\hline 20 & 2,10 & 1,30 & 2,57 & $\mathrm{~A}$ & $\mathrm{~A}+$ & 43 & 1,30 & 0,50 & 1,85 & $\mathrm{~A}$ & $\mathrm{~A}+$ \\
\hline 21 & 1,30 & 0,50 & 8,26 & $\mathrm{~A}$ & $\mathrm{~A}-$ & 44 & 1,45 & 0,65 & 1,10 & $\mathrm{~A}$ & $\mathrm{~A}+$ \\
\hline 22 & - & - & - & - & - & 45 & 1,17 & 0,97 & 8,83 & $\mathrm{~A}$ & $\mathrm{~A}-$ \\
\hline 23 & 1,10 & 0,30 & 2,42 & $\mathrm{~A}$ & $\mathrm{~A}+$ & 46 & 1,52 & 0,72 & 5,31 & $\mathrm{~A}$ & $\mathrm{~A}$ \\
\hline
\end{tabular}

Nota: PLOS [peatones/min/m], PLOS HCM: PLOS Manual de Capacidad de Carreteras y PLOS CL: PLOS manual de la ciudad de Londres

\section{Conclusiones y recomendaciones}

Se logró analizar la movilidad peatonal en el centro de Guadalupe de Goicoechea. Se caracterizó a los usuarios de las facilidades peatonales y sus flujos. Se establecieron los diferentes niveles de servicio peatonales para las aceras analizadas, también se identificaron las aceras con mayor volumen de peatones en la zona de estudio. Estos resultados pueden ser usados como insumos para elaborar un plan maestro de movilidad peatonal en la zona de estudio. 
Analizar la movilidad peatonal es posible; sin embargo, se requiere de una gran cantidad de recursos para poder realizar dicha tarea, dados los cambios en los volúmenes peatonales en espacios relativamente reducidos.

Se aclara que los conteos realizados no son conteos de peatones propiamente, más bien son conteos de viajes. Esto se debe a que si el peatón vuelve a cruzar la línea imaginaria establecida por el sensor, o por la persona realizando el conteo en el periodo de análisis, se vuelve a contar (City of Vancouver, 2015).

Las variaciones espaciales son los cambios en el volumen de peatones por el entorno en el cual se encuentra el punto de estudio. Así que estas variaciones se relacionan con el uso del suelo, cercanía a paradas de transporte público y la presencia de sitios que atraen y generadores de peatones, viéndose afectado por locación donde se encuentran (Aultman-Hall, Lane, \& Lambert, 2009).

La población peatonal es muy variable, se encontró peatones de todas las edades especialmente jóvenes. Después de los 55 años, se observó una disminución de peatones conforme aumenta la edad de los mismos.

Los peatones en su mayoría realizan viajes intermodales (79\%); de los peatones que realizan viajes intermodales un $86 \%$ usan el autobús. De acuerdo con las respuestas obtenidas, los dos principales motivos para caminar en la zona son realizar mandados e ida y regreso del trabajo.

Los peatones encuestados viven principalmente en Goicoechea (71 \%); en los distritos de Guadalupe, Ipís, Purral y Mata de Plátano, los cuales son los más poblados del cantón. Un $83 \%$ de los encuestados trabaja en la provincia de San José, y un 78 \% trabaja Goicoechea y sus alrededores. El otro $5 \%$ de los peatones trabaja en lugares conectados a Guadalupe por las rutas de buses. Así que los principales generadores de peatones son: las zonas residenciales, zonas de trabajo y el transporte público.

Para los peatones es más importante el tiempo que la distancia recorrida, de acuerdo con los datos obtenidos los peatones usualmente caminan entre 5 y 35 minutos.

Se les preguntó a los peatones qué los motivan o desmotivan a caminar, se destacan cuatro variables que motivan a los peatones a caminar según las respuestas de la encuesta: árboles y zonas verdes, iluminación, semáforos peatonales y sitios de descanso. 
Ana Fernández-Garza, Henry Hernández-Vega. Estudio de la movilidad peatonal en un centro urbano: un caso en Costa Rica

Los lugares transitados por muchos peatones, generan opiniones divididas entre seguridad y comodidad. Respecto a la percepción de seguridad, se resalta la importancia de conocer el volumen que transita y la capacidad del espacio peatonal para así brindar a los peatones los anchos de circulación que necesitan en cada acera. Los cuales van a atraer a más peatones dando al lugar una percepción de seguridad.

Un $13 \%$ de las aceras estudiadas tiene un ancho menor a 1,20 metros, un $33 \%$ tienen un igual a 1,20 metros y menor a 1,50 metros, y por último un $54 \%$ tienen un ancho mayor o igual a 1,50 metros. Solo un 33 $\%$ de las aceras en estudio tienen un ancho de circulación menor al ancho ocupado por el peatón de diseño ( 0,60 metros).

Al evaluar el nivel de servicio peatonal, las aceras obtuvieron buenas calificaciones. Se resaltan que en $\operatorname{los}$ puntos $7,9,11,21$ y 45 tiene un flujo de ancho unitario mayor a 8,0 peatones $/ \mathrm{min} / \mathrm{m}$ con calificaciones entre Ay B-. El punto 11 obtuvo una calificación de B- y el punto 21 de A-, con zonas de circulación de 0,40 y 0,50 metros respectivamente.

Los puntos con mayor TPDS se ubican principalmente en la avenida 31 cercanos a paradas de buses, comercio, restaurantes y centros médicos. La dirección del flujo en la avenida 31 es de oeste a este, siendo mayor que el flujo en las direcciones norte - sur. Los puntos 20, 21 y 25 no se encuentran en la avenida 31 , pero tiene alto volumen de peatones por estar cercanos a paradas del transporte público y ser un lugar de importancia para el cantón como es la ferretería Grupo Guadalupano. Los puntos con menor TPDS son: el punto 5 y el 44 (ubicados cercanos a las zonas residenciales del distrito).

Los mayores volúmenes peatonales se dan en la mañana, empezando a las cuatro de la mañana y tendiendo a cero a las once de la noche, teniendo un periodo usual de cero peatones de 11:00 p.m. a 4:00 a.m.

La variación horaria en los diferentes puntos se puede resumir en cuatro tipos:

- $\quad$ Los que exhiben un periodo pico en la mañana, es encuentran cercanos a escuelas y servicios médicos (Instalaciones de INS y Clínica Jiménez Núñez).

- Los que tienen dos periodos picos uno en la mañana y otro en la tarde, continuos a instituciones de servicio, comercios y residencias. 
- Los que presentan un periodo constante durante el día, ubicados cercanos a venta de comidas y comercio.

- Los que muestran tres periodos picos en la mañana, mediodía y en la tarde, se encuentra entre la zona comercial y residencial.

- Los que enseñan un periodo pico en la tarde, se ubican principalmente cercanos a paradas de buses y zona residencial.

$\mathrm{Al}$ analizar las diferentes horas picos de los diferentes grupos se determina que los periodos de conteo entre semana deberían ser de 5:00 a.m. a 10:00 a.m., 12:00 p.m. a 2:00 p.m. y de 4:00 p.m. a 6:00 p.m.

El comportamiento horario del día sábado en los diferentes puntos muestra un alto volumen de peatones de 8:00 a.m. a 2:00 p.m., con un pico especialmente alto de 10:00 a.m. a 12:00 p.m. Los domingos presentan mayor variabilidad que los días sábados y entre semana, pero presenta un alto volumen en las tardes y noches.

Se tiene una diferencia en el comportamiento entre semana y fin de semana, pero especialmente entre el sábado y el domingo. La estación permanente (punto 9) obtuvo un TPDS entre semana de 1776 , un TPDS para el sábado de 1409 y un TPDS para el domingo de 868, siendo tres cuartos el volumen del sábado comparado con el de entre semana y un medio el del domingo, al compararlo con el de entre semana.

La variación semanal muestra como el volumen de peatones va aumentado de lunes a jueves, a partir del cual empieza a disminuir los días viernes, sábado y domingo. En la zona en estudio se encontró mayor variabilidad los días lunes, jueves y viernes, así que los días ideales para ser conteos son: martes y miércoles, especialmente el miércoles.

Al buscar incentivar la movilidad peatonal se consideran pertinentes las siguientes recomendaciones:

- Generar campañas para promover el caminar.

- Al buscar promover el caminar, se debe buscar mejorar el transporte público. Estos dos medios van de la mano.

- Las municipalidades deben invertir en arborizar y promover espacios públicos con zonas verdes, ya que son uno de los principales factores que motivaría a caminar a las personas. Además, es recomendable que las municipalidades no dependan únicamente de la iluminación 
Ana Fernández-Garza, Henry Hernández-Vega. Estudio de la movilidad peatonal en un centro urbano:

un caso en Costa Rica

pública, sino que cuente con la ayuda de las luces de los comercios y evitar las cortinas metálicas por completo que no permiten visualizar las vitrinas de estos. También promover espacios público limpios, ya que la suciedad es el principal desmotivador para caminar.

En cuanto a la infraestructura peatonal analizada en el sector, se recomienda:

- Evaluar los resultados de las calificaciones otorgadas por el manual de la ciudad de Londres y las utilizadas en el Manual de Capacidad de Carreteras, al analizar el nivel de servicio peatonal.

- Realizar un análisis de infraestructura y condición de las aceras, como complemento al análisis de monitoreo que se esté realizando.

- Eliminar los obstáculos en los puntos: parque público, avenida 31 (9), casa vieja, continua a las instalaciones del INS (14), en el medio de la cuadra con tapia verde (23), en la acera frente del Restaurante Bar Sport Grill (26), en la acera enfrente de la peluquería Sebastián (27) y en la parada de taxi de Novacentro (45). Además, realizar una adecuación de toda la infraestructura peatonal para que una persona con discapacidad o que lleve un coche pueda movilizarse libremente sin necesidad de invadir la vía de los vehículos.

\section{Referencias}

Ángel, J. (s.f.). Medellín, ciudad y movilidad. Alcandía de Medellín, Secretaría de Transporte y Tránsito de Medellín, Medellín. Recuperado el 27 de Agosto de 2016, de Cities for mobility: http://www.cities-for-mobility. net/documents/wc07/WSD_110607_JoseFernandoAngelPerez.pdf

Aultman-Hall, L., Lane, D., \& Lambert, R. (2009). Assessing impact of weather and season on pedestrian traffic volumes. Transportation Research Record: Journal of the Transportation Research Board(2140), 35-43. doi:10.3141/2140-04

Azmi, D. I., Karim, H. A., \& Amin, Z. M. (19 de Diciembre de 2012). Comparing the walking behaviour between urban and rural residents. Procecia-Social and Behavioral Sciences, 68, 406-416. Recuperado el 19 de Abril de 2016, de ScienceDirect: http://www.sciencedirect. com/science/article/pii/S1877042812057205 
Barquero, L., \& Blanco, M. (1993). Estudio de la capacidad de servicio de calles, aceras y estacionamientos, dentro de la ciudad universitaria Rodrigo Facio y accesos principales. San José: Trabajo Final de Graduación, Universidad de Costa Rica.

Bull, A. (2003). Congestión del tránsito. El problema y cómo enfrentarlo. Santiago, Chile: Comisión Económica para América Latina y el Caribe.

City of London. (2013). Aldgate Gyratory pedestrian analysis for the City of London. Reporte final, City of London, Transport and Streets, London. Recuperado el 08 de Julio de 2016, de https://www.cityoflondon. gov.uk/services/transport-and-streets/transport-planning/transportprojects/aldgate-area/Documents/02-pedestrian-analysis.pdf

City of Vancouver. (Mayo de 2015). 2013 pedestrian volume and opinion survey. Recuperado el 08 de Junio de 2016, de City of Vancouver: http://vancouver.ca/files/cov/report-2013-pedestrian-volume-opinion-survey.pdf

Department of Transportation. (27 de Agosto de 2016). Thinking about pedestrian from the star - creating pedestrian - friendly communities. Obtenido de ehawaii.gov: https://hidot.hawaii.gov/highways/files/2013/07/ Pedest-Tbox-Toolbox_1-Thinking-about-Peds-from-the-Start.pdf

Espinoza, J. L. (2015). Estudio de Movilidad Ciclista en los Distritos del Cantón de Puntarenas con Mayor Cantidad de Ciclista Involucrados en Colisiones. Proyecto de Graduación grado de Licenciatura, Universidad de Costa Rica, Escuela de Ingeniería Civil, Montes de Oca. Recuperado el 20 de Abril de 2016.

Figliozzi, M., Johnson, P., Monsere, C., \& Nordback, K. (2014). A methodology to characterize ideal short-term counting conditions and improve AADT estimation accuracy using a regression-based correcting function. Journal of Transportation Engineering, 140(5), 4-14. Recuperado el 20 de Abril de 2016, de http://ascelibrary.org/ doi/abs/10.1061/(ASCE)TE.1943-5436.0000663

Figliozzi, M., Monsere, C., Nordback, K., Johnson, P., \& Blanc, B. (2014). Design and implementation of pedestrian and bicycle - Specific data collection methods in Oregon. Final, Oregon Department of Transportation, Oregon. Recuperado el 20 de Abril de 2016, de http:// www.oregon.gov/ODOT/TD/TP_RES/ResearchReports/SPR754_ Ped_and_Bicycle_PilotStudy.pdf. 
Ana Fernández-Garza, Henry Hernández-Vega. Estudio de la movilidad peatonal en un centro urbano:

un caso en Costa Rica

Garricho, C. Aguilar, A., Brambila, C., \& Sabrino, J. (Noviembre de 2014). Hacia una cultura de las ciudades sostenibles. IV Congreso de la Asociación Latinoamerica de Población (Lima, agosto, 2014), Fondo de Población de Naciones Unidades (UNFPA). Recuperado el 27 de Agosto de 2016, de Fondo de Población de las Naciones Unidades: http://www.unfpa.org.mx/publicaciones/ciudadessostenibles.pdf

Gehl, J. (2010). Cities for people. Washington, DC, Estados Unidos: Island Press. Gehl, J., Westermark, E., Rison, J., Mortensen, H., Adams, R., \& Rorbech, J. (2009). Worls Class Streets: Remaking New York City's Public Realm. New York City, Department of Transportation, New York. Recuperado el 27 de Agosto de 2016, de NYC: http://www.nyc.gov/ html/dot/downloads/pdf/World_Class_Streets_Gehl_08.pdf

Grifin, G., Nordback, K., Gotschi, T., Stolz, E., \& Kothuri, S. (Marzo de 2014). Monitoring bicyclist and pedestrian travel and behavior: Current research and practice. Transportation research circular (EC183), 1-32. Recuperado el 19 de Abril de 2016, de Missoula, MT: www.ci.missoula.mt.us/DocumentCenter/View/28036

Hughes, R. (2002). A continuun theory for the flow of pedestrian. Transportation Research Part B: Methdological, 36(6), 507-535. Recuperado el 19 de Abril de 2016, de http://www.sciencedirect.com/ science/article/pii/S0191261501000157

Kittelson \& Associates, Inc, Ryan Snyder Associates \& Los Angeles County Bicycle Coallition. (2013). Conducting bicycle and pedestrian counts: A manual for jurisdictions in Los Angeles county and beyond. Manual, The sounthern california association of goverments, Los Angeles. Recuperado el 27 de Abril de 2016, de https://media. metro.net/.../bikes/bikecounttrainingmanual_2.pdf

Lachapelle, U., \& Noland, R. (15 de Febrero de 2012). Does the commute mode affect the frequency of walking behavior? The public transit link. Transport policy , 21, 26-36. Recuperado el 19 de Abril de 2016, de http://www.sciencedirect.com/science/article/pii/S096707

Laing, J., Turner, S., \& Carr, A. (2009). Pedestrian plannig and design guide. Guía, NZ Transport Agency, National Office, Wellington. Recuperado el 8 de Junio de 2016, de New Zealand Transport Agency: https://www.nzta.govt.nz/.../pedestrian-planning-guide/.../pedestrian-planning-guide.pdf 
Lindsey, G., Nordback, K., \& Figliozzi, M. A. (2014). Institutionalizing bicycle and pedestrian monitoring programs in three states: Progress and challenges. 93rd Annual Meeting of the Transportation Research Board Washington, DC, 1-22. Recuperado el 19 de Abril de 2016, de http://trrjournalonline.trb.org/doi/abs/10.3141/2443-15

Magaña, J. (2014). Determinación de patrones típicos de distrubución temporal de tránsito en Costa Rica. Proyecto de graduaciòn para el grado de licenciatura, Universidad de Costa Rica, Escuela de Ingeniería Civil, San José, Costa Rica. Recuperado el 27 de Abril de 2016.

Medina, S. (Marzo de 2012). Transformando la movilidad urbana en México. Instituto de Políticas para el Transporte y el Desarrollo (ITDP México), Proyecto de estrategias de reducción del uso del automovil en ciudades mexicanas. Mexico: Grupo Fogra, S.A. de C.V. Recuperado el 27 de Agosto de 2016, de ITDP México: http://mexico. itdp.org/wp-content/uploads/Transformando-la-movilidad-urbanaen-Mexico.pdf

Milligan, C., Poapst, R., \& Montufar, J. (2013). Performance measures and input uncertainty for pedestrian crossing exposure estimates. Accident Analysis \& Prevention, 50, 490-498.

Millward, H., Spinney, J., \& Scott, D. (Abril de 2013). Active - transport walking behavior: destinations, durations, distances. Journal of Transport Geography, 28, 101-110. Recuperado el 20 de Abril de 2016, de ScienceDirect: http:/www.sciencedirect.com/science/ article/pii/S096669231200289X

Molloy, S., Kirkpatrick, A., \& Hull, D. (6 de Noviembre de 2013). Pedestrian mobility plan (PW 13078) - (City Wide). City of Hamilton, Pubic Works Department, Hamilton. Recuperado el 20 de Abril de 2016, de Hamilton: http://www2.hamilton.ca/NR/rdonlyres/517842777BB3-4DC7-8E6D-09404410841C/0/Nov0671PW13078.pdf

Ortúzar, J. d. (2000). Modelos de demanda de transporte (Segunda ed.). Mexico, D.F., Mexico: Alfaomega. Recuperado el 3 de Agosto de 2016 Pérez, M., \& Alvarado, R. (2004). Aceras, Peatones y Espacio Público. Serie Ordenamiento Territoral: $N^{\circ} 5$, Dirección de Gestión Municipal, Sección de Gestión Municipal . Recuperado el 04 de Abril de 2016, de A convivir: https://bibliotecapromocion.msp.gob.ec/greenstone/collect/promocin/index/assoc/HASH7642.dir/doc.pdf 
Ana Fernández-Garza, Henry Hernández-Vega. Estudio de la movilidad peatonal en un centro urbano: un caso en Costa Rica

Poapst, R., \& Montufar, J. (Mayo de 2012). Pedestrian traffic data collection: State of the practice in Canada and U.S. CITE Annual Conference, May 2012, University of Manitoba, Department of Civil Engineering. Recuperado el 23 de Agosto de 2015, de Canadian Institute of Transportation Engineers: http://www.cite7.org/conferences/compendium/2012_Pedestrian_PedestrianTrafficDataCollection_StatePracticeCanadaUS.pdf

Roberts, M., \& Black, T. (Setiembre de 1997). Pedestrian facilities guidebook. Manual, Otak, Inc, Washington. Recuperado el 27 de Abril de 2016, de WSDOT Washington State Department of Transportation: http://www. wsdot.wa.gov/publications/manuals/fulltext/m0000/pedfacgb.pdf

Royal, D., \& Miller - Steiger, D. (Agosto de 2008). Volumen III: Methods Report National Survey of Bicyclist and Pedestrian Attitudes and Behavior. Obtenido de: http://www.nhtsa.gov/DOT/NHTSA/Traffic\%20 Injury\%20Control/Articles/Associated\%20Files/810973.pdf

Ryan, S., \& Lindsey, G. (Febrero de 2013). Counting bicyclists and pedestrian to inform transportation planning. Investigación breve, Robert Wood Johnson Fundation, San Diego. Recuperado el 27 de Abril de 2016, de School of Public Affairs, San Diego State University: http:// ipua.sdsu.edu/docs/ALR_Brief_Bike-PedCounts_Feb2013.pdf

Sauter, D., Pharoah, T., Tigt, M., Martinson, R., \& Wedderburn, M. (2015). International walking data standard. Pre-final version, Measurin Walking - Walk21. Recuperado el 26 de Septiembre de 2016, de: files.designer.hoststar.ch/hoststar10546/file/1-international_walking_data_standard_version_aug_2016.pdf

Serrano, V. (1986). Análisis del flujo peatonal en el área de San José. San José: Trabajo Final de Graduación Universidad de Costa Rica.

Sepúlveda, B., Cañez, J., Garduño, J., Media, S., Orozco, M., Padilla, X., . . . Veloz, J. (s.f.). Hacia una Estrategia Nacional Integral de Movilidad Urbana. Instituto de Políticas para el Transporte y el Desarrollo. Recuperado el 27 de Agosto de 2016, de ITDP: http://mexico.itdp. org/wp-content/uploads/Movilidad-Urbana-Sustentable-MUS_.pdf

South Alabama Planning Commission. (s.f.). Bicycle and Pedestrian Survey. Recuperado el 19 de Abril de 2016, de Bike Ped Planning: http://www. mobilempo.org/Bicycle\%20Pedestrian\%20Comprehensive\%20Plan/ Bicycle\%20and\%20Pedestrian\%20Survey\%20Results.pdf 
The Gilmore Research Group. (Mayo de 2007). Public attitude survey of bicycle and pedestrian planning. Final, Wilbur Smith Associates, Bellevue. Recuperado el 08 de Junio de 2016, de WSDOT: https:// www.wsdot.wa.gov/NR/rdonlyres/E09B18F2-8301-4205-A73FFBD3D27DBB98/0/SurveyReport.pdf

Torres, M. (2016). Estudio de la movilida ciclista en los distritos de San Francisco, Guadalupe y Quebradilla del cantón de Cartago, y los distritos de Tobosi y Tejar del cantón de El Guarco. Trabajo Final de Graduación grado de Licenciatura, Universidad de Costa Rica, Escuela de Ingeniería Civil, Montes de Oca. Recuperado el 20 de Abril de 2016.

Transportation Research Board. (2010). Highway Capacity Manual. National Academy of Sciences. Washington, DC: The National Academies of Sciences Engineering and Medicine.

Umaña, H. (18 de Septiembre de 2015). Conteos intersecciones Guadalupe. (A. G. Fernández, Entrevistador) San José, Costa Rica.

Vega-Padilla, V., \& Hernández-Vega, H. (2017). Metodología para evaluación de aceras como parte de la gestión de activos urbanos en Costa Rica. Tecnología MOPT, 25.

Yang, H., Ozbay, K., \& Bartin, B. (11 de Julio de 2010). Investigating the performace of automatic counting sensors for pedestrian traffic data collection. Proceedings of the 12th World Conference on Transport Research, 1115, 1-11. Recuperado el 20 de Abril de 2016, de http:// www.wctrs-society.com/wp/wp-content/uploads/abstracts/lisbon/ selected/02147.pdf 